\title{
When Should Customers Control Service Delivery? Implications for Service Design
}

\author{
Ioannis Bellos \\ School of Business, George Mason University, Fairfax, VA 22030, ibellos@gmu.edu \\ Stylianos Kavadias \\ Judge Business School, University of Cambridge, Cambridge, United Kingdom, s.kavadias@jbs.cam.ac.uk
}

What do a Mongolian stir-fry restaurant and a medical lab providing home testing solutions have in common? They are both innovative services that base their success on customers controlling part of the service delivery. These providers allow service tasks to be performed by the customers as a means of shaping the overall experience and not strictly as a means of "outsourcing" the service. Motivated by such practices, we explore whether and how should providers allocate the control of different tasks of their service to the customers. We model services as multi-step processes with each step affecting customers' experience at other steps. At certain steps the provider may hold an "expert" role and be more capable of performing than the customers, whereas at other steps she holds an "administrative" role and is less capable of performing than the customers. We distinguish between routine services, where the service outcome must conform to standardized specifications, and non-routine services, where the value of the service outcome relies on subjective dimensions. We show that the optimal design is determined by an economically intuitive rule whereby the provider controls the steps based on the marginal benefit she can derive compared to self-service. For routine services, this rule translates to managing "blocks" of steps because the provider benefits from containing the volatility of the experiences across the service even when this implies the provision of service steps with a negative marginal benefit, i.e., steps which she is less capable of performing than the customers. Instead, in non-routine services providers should focus on the value advantage they can ensure through a "core provision" even if this implies forgoing control of steps for which they are more capable of performing than the customers and from which they can derive positive marginal benefit. This implies that in non-routine services the provider exercises more control up to a certain process length; beyond that she delegates more steps to the customers. When customers differ in their abilities to perform the different steps, the provider may offer a service line. Service lines facilitate better segmentation than a single service offering, but their economic benefit exhibits an inverted "U-shaped" relationship with respect to the number of steps that a service comprises. Finally, we find that competition between two providers who differ in their capabilities to perform a service results in service design differentiation where the more capable provider offers a higher-end "focused service" against a lower-end "super-service" offered from the less capable provider.

Key words: customer experience, service process, service line design, service competition 


\section{Introduction}

Customer engagement during service delivery has been long recognized as a "mixed blessing" for service providers (Sasser 1976). On the positive side, customers can be an indirect source of labor that results in cost savings, but on the negative side, they may also introduce variability because they may interact with the provider in complex and uncertain ways. Advances in technology have helped providers to capitalize on the positive side. For instance, self-checkout terminals have allowed providers to capture non-negligible operational cost savings. However, viewing customer involvement as merely a cost-saving strategy is rather limiting and often results in poorer service outcomes (Moon and Frei 2000). The reason is that such involvement rarely results in immediate benefits for the customer, who, let us not forget, exerts the effort (Frei and Morriss 2012). Building on this observation, we posit that whether a service task is controlled by the customer or the service provider, is not strictly a means of achieving operational cost savings. Instead, it offers a means of shaping the overall customer experience. Recent examples of innovative services indicate how customer involvement is an opportunity to create customer value:

Dining Services. In midtown Toronto, Canada, Genghis Khan Mongolian Grill encourages their customers to be creative and enjoy their own food creation. In particular, customers choose from a wide variety of ingredients and prepare a combination of their own inspiration. Then, they let the chefs assume the cooking responsibility. This service blueprint lies in contrast to that of another equally unconventional restaurant: the Gaslamp's Steak Place in San Diego, CA. This well-known Californian steak lounge lets the customers grill their own selection of steak and thereby delegates the food preparation responsibility to them. Several miles away, in San Francisco, CA, Eatsa only prepares the food and delegates the rest of the service tasks to their customers. Eatsa's customers select and place their orders on touchscreen tablet computers, and proceed to wait before a wall of glass cubbies. Once their order is ready, it is placed in one of the cubbies which light up with the name of the customer, who then picks up the order. Finally, Blue Apron's online service controls all tasks except for cooking. It selects the food ingredients, provides recipes/cooking instructions, and delivers everything to the customer who then assumes the cooking responsibility at their own space.

Healthcare Services. Testing the blood sugar levels of a diabetic patient no longer requires a visit to the doctor or the lab. Most patients can easily obtain and use self-test kits 
(WebMD 2018). More interestingly though, new services allow for customers to exercise similar control over testing, even for conditions that require more complicated specimen analysis. For instance, Cologuard (specializing on the detection of colon cancer) or myLab Box (specializing on the detection of sexually transmitted diseases) allow customers to be tested for different health conditions by mailing their samples to the provider's lab. Similarly, the customers of SmileDirectClub initially submit an online photo assessment of their dental structure. After a licensed dental professional reviews this assessment, the customer receives a kit with instructions on how to create his dental impressions. These impressions are mailed to the provider, and the lab creates the customized dental aligners which are then shipped to the customer. Throughout the treatment, the customer regularly uploads pictures so that the provider monitors the overall progress.

These diverse examples showcase how the customer's role can be leveraged in practice by innovative service providers. The same examples also reveal that determining how to manage this role is far from a straightforward decision. Even in similar contexts (e.g., dining services), different providers implement different designs when it comes to enabling customer control over various service tasks. The differences stem from the challenges associated with the customers' involvement in service delivery. For instance, not all customers have the same abilities to perform the different tasks (e.g., cooking) of a service process. Such heterogeneity can create a tension as low-ability customers may prefer to relinquish control of the service tasks to the provider, but high-ability customers may actually enjoy exercising more control over the service tasks.

Regardless of their ability level and independently from the nature of the service, customers may prefer to relinquish control if the provider can perform the service more reliably than them. However, such reliability benefits may vanish if the provider control necessitates more inter-personal interaction. For instance, explaining special meal or cooking preferences to a waiter may result in a more variable experience than when the customer picks and cooks his own selection of ingredients. ${ }^{1}$ Finally, the fact that services comprise multiple tasks (i.e., service steps) implies that the experience that customers derive at one service step may affect the experience they derive at other steps.

\footnotetext{
${ }^{1}$ The higher experiential variability can also be attributed to "social frictions" during the interaction of a customer and a service provider. For instance, a customer may feel that he is subject to negative social judgement when he places a complicated request or even when he orders high-calorie food; see Goldfarb et al. (2015).
} 
In this paper, we answer the question of how providers should allocate the control of the different service tasks to the customers. We consider a monopolist provider who decides which steps of the service process to "delegate" to the customers. We define the value that customers derive at each service step to comprise two parts: i) a functional (systemic) part and ii) an idiosyncratic (emotional/experiential) part. The customer value realized at the different steps might be correlated and the customers, all else being equal, prefer less variable experiences. Furthermore, customers may differ in their ability to perform the service steps.

The difference between the functional value that customers derive from a service step when it is performed by the provider versus themselves defines the provider's expertise advantage at that step. In certain steps, the service provider has an "expert" role and is more capable of delivery than the customers. At such steps, her expertise advantage is positive, and the customers derive larger functional value when the provider controls the steps. In other steps, the provider holds an "administrative" role and might be less capable of satisfactory delivery. There, her expertise advantage is negative, and the customers derive larger functional value from self-performance.

Which party controls a service step also affects the idiosyncratic part of the value derived by the customer. In particular, the value contribution of the idiosyncratic part may increase or decrease depending on the party that assumes responsibility for a service step. This distinction between a higher or lower contribution from the idiosyncratic part finds an intuitive conceptual mapping to the type of the service. We distinguish between routine and non-routine services. In routine services, the value of the service outcome must conform to standardized specifications, and in that context, a task delivered by the provider might also ensure conformity (i.e., less variability). In non-routine services, the service outcome is evaluated along more intangible dimensions, like subjective taste. Hence, if the customer does not have direct control of a service step, this may amplify the variability of how he evaluates the outcome of the step.

Our analysis characterizes a practical and economically intuitive rule that prioritizes which steps should be controlled by the service provider based on their marginal benefit to the provider. This benefit is determined by the extent to which the provider's expertise advantage at a service step outweighs the total cost of providing the step. Although this rule is independent of the service context, the resulting optimal design differs significantly 
depending on the type of the service. In routine services, the provider prefers to "overcontrol." She controls even steps for which she is less capable of performing compared to the customers; steps with a negative marginal benefit. This happens to ensure the overall performance of the service outcome through less variability. On the contrary, in non-routine services, the provider focuses focus only on the subset of steps for which she holds a clear and sizeable expertise advantage forgoing steps with small but positive marginal benefit. Through such designs, she ensures high outcome delivery while mitigating the possible detrimental effects of variability across the service steps.

Furthermore, we investigate how service characteristics affect the provider's optimal design. For instance, in routine services, the provider controls more steps if the service comprises a large number of steps. Yet, in non-routine services, this effect is non-linear. The provider exercises more control for longer processes, but beyond a certain length, she decreases the number of steps she controls. Regardless of the type of the service, however, the provider always derives higher profit from services where her expertise advantage varies significantly across the different service steps. Any differences in the provider's expertise advantage across the different steps stem from differences in the provider's expertise, the customers' capability and/or the cost of the service steps. Hence, in practical terms, our finding implies that all else being equal, the provider prefers service processes that comprise dissimilar, in terms of cost or contribution to the customer value, steps.

Our paper also provides insights regarding the effect of heterogeneity in the customers' capability to perform the different service steps. We consider the case where a service provider covers the needs of a heterogeneous market by offering a service line with two different services. We find that the economic benefit of introducing a service line exhibits an inverted "U-shaped" relationship with respect to the number of the service steps. Namely, the economic benefit from offering a service line is less pronounced for services that comprise a small or large number of steps. Thus, for significant deployment costs (e.g., due to acquiring new facilities and equipment, as is often the case because services cannot be inventoried), a service line may be economically non-viable. Hence, the classic benefit of better market segmentation due to a service line might be critically traded-off by characteristics of the service such as its number of steps. This result is a novel insight beyond the traditional outcomes of product line design. 
Via numerical methods, we also tease out the effect of competition on service design. We consider the case of two competing providers who differ in their ability to perform the service steps. Competition induces more differentiated service offerings: a "focused service" where the high-capability provider exercises control of key service tasks only and a "super-service" where the low-capability provider assumes control of a large number of service tasks. Interestingly, although in a monopolistic setting a higher-capability provider controls more service steps to capitalize on her expertise advantage throughout the service process, under competition we find that the higher-capability provider limits her control of the service process.

\section{Literature Review}

Our focus on customer involvement as a design decision echoes a growing literature stream, which adopts the broader perspective of customers as value co-creators (Prahalad and Ramaswamy 2000, Thomke and Von Hippel 2002). The primary focus of this stream, however, has been on the design of tangible products (see Franke et al. 2010, Basu and Bhaskaran 2018 and references therein), which due to their tangible nature, are characterized by a smaller degree of uncertainty around the customer experience than services (Zeithaml et al. 1985).

A recent stream of research has investigated the customers' role in the delivery of services and how it can be enabled by technology (Scherer et al. 2015). The focus of these studies has been primarily on the technology-mediated interactions between customers and service providers (Froehle and Roth 2004), taking place through the use of self-service technologies (SSTs; see Buell et al. 2010, Campbell and Frei 2010, Ba et al. 2010, Kumar and Telang 2012). In this stream of research, the decision to engage the customer is based mainly on the potential cost savings that typically ensue from the engagement of SSTs in specific steps of the service process. Still, these papers do not account for the holistic customer experience across all points of contact between the provider and the customer during the service delivery. The customers' role has also been examined in B2B service delivery contexts (e.g., consulting projects; see Xue and Field 2008, Roels et al. 2010, White and Badinelli 2012, Karmarkar and Roels 2015, Demirezen et al. 2016). In such contexts, however, the design decisions revolve mainly around the design of formal contractual agreements between a client and a service provider. 
Our research is closer in spirit to the research of Soteriou and Hadjinicola (1999) and Bellos and Kavadias (2017), who both consider services that comprise multiple steps. Soteriou and Hadjinicola (1999) focus on the optimal budget allocation across the service steps but assume no dependence between steps. Bellos and Kavadias (2017) borrow the same conceptual approach to service design as a follow-up to our study and determine the optimal provider effort allocation across the different service steps. Neither of these papers offers actual insights regarding which service steps should be performed by the customer. Furthermore, both papers assume that customers are homogeneous and that the provider always offers a single service.

Our paper offers a framework that analytically formalizes novel service design practices. We contribute to the nascent field of service design by explicitly accounting for the multistage nature of services, the inherent variability in the customer value, and the fact that the customer value at one step may be affected by the value at a different step. Our framework applies to a multitude of service contexts and prescribes design guidelines based on a practical typology: that of, routine vs. non-routine services. Furthermore, we account for the fact that the market may comprise customers with different abilities to engage during the service delivery, and we analyze the provider's incentives to design a line of differentiated services. To our knowledge, this is the first paper to offer insights into the design of service lines.

\section{The Model}

In this section, we introduce our model. A monopolist service provider determines the optimal design of their offering: the price to charge and the steps of the service process to control or delegate to the customers.

\subsection{The Service Process}

We formalize a service process as a set $\mathcal{J}=\{1,2, \ldots, n\}$ of $n$ distinct steps that aim to satisfy a particular objective (e.g., diagnosis, treatment of a health condition) and generate customer value. The value $\tilde{V}_{i}^{e}$ that customers derive at step $i$ depends on the entity $e \in$ $\{P, C\}$ that performs it; $P$ denotes the service provider and $C$ the customer.

Formally, a service is defined by the vector $\mathcal{D}=(\mathcal{P}, \mathcal{C})$ where the provider controls a subset of steps $\mathcal{P} \subseteq \mathcal{J}$ and delegates the rest of them $\mathcal{C}=\mathcal{J} \backslash \mathcal{P}$ to the customers. The exact value that a customer realizes from service $\mathcal{D}$ is given by $\tilde{V}(\mathcal{D})=\sum_{i \in \mathcal{P}} \tilde{V}_{i}^{P}+\sum_{i \in \mathcal{C}} \tilde{V}_{i}^{C}$. The 
first sum captures the value that a customer derives from the subset of steps controlled by the service provider. The second sum expresses the value derived from the steps that the customer performs.

Regardless of whether a step is performed by the customer (henceforth "he") or the service provider (henceforth "she"), the exact customer value cannot be predicted at the stage where the design of the service takes place. For that reason, we define $\tilde{V}_{i}^{e} \doteq V_{i}^{e}+\tilde{\varepsilon}_{e}$, where $\tilde{\varepsilon}_{e}$ follows a general probability distribution $\mathbb{G}\left(0, \sigma_{e}^{2}\right) ; \tilde{V}_{i}^{e}$ is a random variable with a mean $V_{i}^{e}$ and variance $\sigma_{e}^{2}$. The distribution moments of the customer value carry very intuitive meanings: the mean captures the functional part of the customer value at a service step (e.g., subsided symptoms after treatment of a health condition). The variance represents the idiosyncratic, emotional and experiential part of the customer value. It can be interaction/moment-specific, and it determines how customers perceive the functional benefit $V_{i}^{e}$ during a particular interaction instance. For example, a patient who received a successful treatment may still assess the overall service less favorably if he feels he was rushed by the doctor. We refer the reader to Berry et al. (2002) for a more detailed description of these two factors.

Let the functional part at each step $i$ be $V_{i}^{P} \geq 0$ when the step is performed by the provider and $V_{i}^{C} \geq 0$ when the step is delegated to the customer. The value of $V_{i}^{P}$ depends on the provider's expertise and capability to perform the specific step. Similarly, the value of $V_{i}^{C}$ depends on the customer's capability to perform the specific step. ${ }^{2}$ As an example, the value $V_{\text {Registration }}^{C}$ is larger for a technologically savvy customer who can efficiently use an online registration system than for a customer who is less acquainted with the use of technology. The difference $\Delta V_{i} \doteq V_{i}^{P}-V_{i}^{C}$ between the functional components at each step characterizes the provider's expertise advantage over the customer (i.e., the added functional value that the provider contributes by controlling step $i$ ). Although the standard expectation would be that $\Delta V_{i}>0$, as this justifies the need for a service provider in the first place, one could think of service steps where $\Delta V_{i}<0$. This can be the case when, all else being equal, the customer is better, or more efficient, at executing the task at hand than the service provider. For instance, explaining seating preferences to an agent may

\footnotetext{
${ }^{2}$ The value of $V_{i}^{C}$ may also be limited by the extent to which existing technology can facilitate customers in selfperforming a service step. For instance, self-checkout machines at grocery stores are known to have several shortcomings (The Wall Street Journal 2013). Advances in retail automation aim at addressing such shortcomings (The Wall Street Journal 2016).
} 
take more time than self-selecting seats directly through an automated terminal. At such a step, the provider's role is rather administrative than value-adding or expertise-based. This description also captures manifestations of the "IKEA effect," which postulates that some customers may value more the creations for which they exerted more effort; see Norton et al. (2012). Such a higher valuation of the service outcome may also be attributed to the enhanced control that customers perceive when they perform a service task independently (Dabholkar 1991, 1996, Bitner et al. 2000).

Regarding the idiosyncratic component $\tilde{\varepsilon}_{e}$ of the customer value, we assume that each customer-provider interaction varies with respect to its exact value realization and we capture this variance through $\sigma_{e}^{2}$. In fact, we posit that the same customer may enjoy a different dining experience at a specific restaurant even if every time that he visits, he is served by the same waiter, sits at the same table, and consumes the same food at the same time of the day. This "noise" is a unique characteristic of service environments. It arises from the fact that customers are an integral part of the service delivery process (Sampson and Froehle 2006).

To vividly illustrate the roles of $V_{i}^{e}$ and $\sigma_{e}^{2}$ in our model, consider the following setup: a patient seeks diagnosis and treatment. Let us assume that the patient is currently at the Registration step. For a healthcare provider, contemplate the following two design scenarios: i) the patient is supposed to self-check-in and self-report his symptoms and medical history by filling out a set of appropriate forms, or ii) specialized personnel (e.g., a nurse) are responsible for the patient's registration. In the latter case, the presence of a nurse ensures that registration happens in a smooth and timely fashion without unnecessary trial-and-error attempts (e.g., fields missing from the registration form). In our model terminology, this case corresponds to an expertise advantage from the healthcare provider through a higher functional benefit, i.e., $V_{\text {Registration }}^{\text {Nurs }}>V_{\text {Registration }}^{\text {Patient }}$.

In the event that a nurse controls the registration, the idiosyncratic component of the experience can add or subtract to the realized customer value. For instance, depending on the medical condition a patient may perceive the presence of a nurse as intrusive, and for that reason, he may feel uncomfortable to share details of his medical history and symptoms $\left(\tilde{V}_{\text {Registration }}^{\text {Nurse }}<V_{\text {Registration }}^{\text {Nurse }}\right)$. On the other hand, it may also be possible that the patient considers the nurse helpful, friendly, and/or reassuring, and thus finds it easier to provide 
the necessary information $\left(\tilde{V}_{\text {Registration }}^{\text {Nurse }}>V_{\text {Registration }}^{\text {Nurse }}\right)$. Since the patient's self-registration experience is not affected by the nurse, it is reasonable to assume that $\sigma_{\text {Patient }}^{2}<\sigma_{\text {Nurse }}^{2}$.

We assume that the magnitude of $\sigma_{e}^{2}$ depends on the nature of the service. For instance, routine services, where the valuation of the service outcome must conform to standardized specifications (e.g., the time to receive the order in a fast food restaurant), are characterized by smaller values of variability than non-routine services where the service outcome is evaluated along more intangible dimensions (e.g., space ambience, or attitude of the waiter in a gourmet restaurant). We consider $\sigma_{C}=\delta \sigma_{P}$, with $\delta>0$ and $\Delta \sigma \doteq \sigma_{P}-\sigma_{C}=(1-\delta) \sigma_{P}$. We characterize non-routine services by $\Delta \sigma>0$, (i.e., $0<\delta<1$ ), implying that the idiosyncratic component of the customer value is more variable at the steps controlled by the provider (due to more interacting parts; i.e., more sources of experiential variability or social frictions). For routine services, we consider $\Delta \sigma<0$, (i.e., $\delta>1$ ), implying that the idiosyncratic component of the customer value is less variable at the steps controlled by the provider. Hence, in routine services the provider maintains a variability "advantage" over the customers whereas, in non-routine services, she maintains a variability "disadvantage."

The distinct values of $\Delta V_{i}$ and $\Delta \sigma$ allow us a useful classification of different services. Higher (lower) values of $\Delta V_{i}$ imply an expertise advantage (disadvantage) from the provider. Lower (higher) values of $\Delta \sigma$ imply more (less) routine services. In that regard, our model outlines design guidelines contingent on different service contexts. Figure 1 provides examples of service steps in the $\Delta V_{i} \times \Delta \sigma$ space under different service contexts.

Finally, the service steps may generate interdependent, complex effects on the value realization at a specific step. This may be due to the nature of the service tasks or the mere fact that the customer's experience at one step may be biased due to the experience at another step. For instance, a less courteous greeter at a restaurant may adversely affect the customer's interaction with the waiter. Therefore, we consider the realizations of the values $\tilde{V}_{i}^{e}$ across the respective steps of the service process to be interdependent through a common pairwise correlation $\rho \in(0,1) .^{3}$

\footnotetext{
${ }^{3}$ From a technical point of view, we consider an $n \times n$ correlation matrix $\mathbf{P}$ with $\rho_{i, j}=\rho \in(0,1) \forall i \neq j$ and $\rho_{i, j}=1$ $\forall i=j . \mathbf{P}$ is a valid correlation matrix if and only if it is positive semidefinite. It is straightforward to show that the determinants $|\mathbf{P}|>0$ and $\left|\mathbf{P}_{k}\right|>0, \forall k \in\{1, \ldots, n-1\}$, where $\mathbf{P}_{k}$ is the principal submatrix that comprises only the 1 to $k$ rows and columns of $\mathbf{P}$. Hence, $\mathbf{P}$ is positive definite and as such represents a valid correlation matrix (see also Rousseeuw and Molenberghs 1994).
} 
Figure 1 Service steps in the $\Delta V_{i} \times \Delta \sigma$ space.

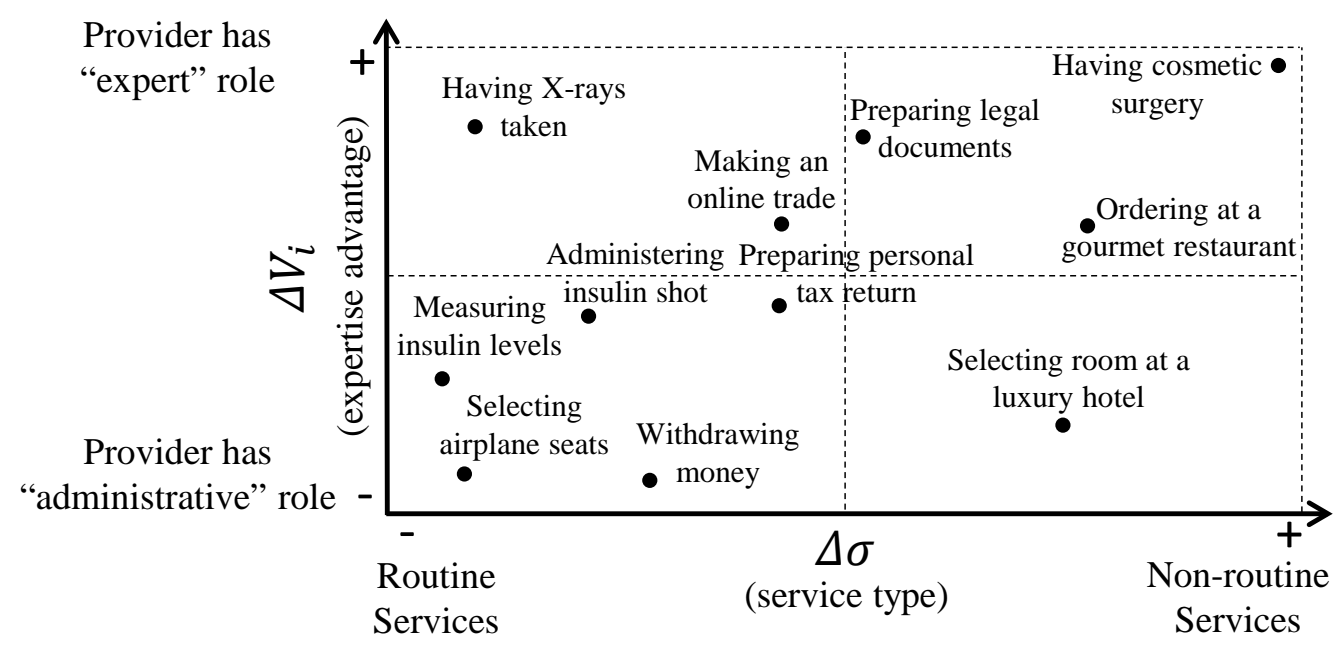

Note. The provider's expertise advantage is characterized by $\Delta V_{i} \doteq V_{i}^{P}-V_{i}^{C} \forall i \in \mathcal{J}$. The type of the service is characterized by $\Delta \sigma \doteq \sigma_{P}-\sigma_{C}=(1-\delta) \sigma_{P}$, where $\delta \in(0,1)$ for non-routine services and $\delta>1$ for routine services.

\subsection{Service Design Decisions}

The service provider makes the irreversible decision of which steps to control/delegate to the customers and also sets the price for the service. The customers observe the provider's choices and determine whether receiving the service is beneficial to them. We continue by formulating the customer's decision.

\subsection{The Customer's Decision}

The overall value that customers derive from going through the entire service process is a random variable $\tilde{V}(\mathcal{D})=\sum_{i \in \mathcal{P}}\left(V_{i}^{P}+\tilde{\varepsilon}_{P}\right)+\sum_{i \in \mathcal{C}}\left(V_{i}^{C}+\tilde{\varepsilon}_{C}\right)$, with mean $V(\mathcal{D})=\sum_{i \in \mathcal{P}} V_{i}^{P}+\sum_{i \in \mathcal{C}} V_{i}^{C}$, and variance $\sigma^{2}(\mathcal{D})=\sum_{i \in \mathcal{P}} \sigma_{P}^{2}+\sum_{i \in \mathcal{C}} \sigma_{C}^{2}+$ $\rho \sum \sum_{i \neq j \in \mathcal{J}}\left(\mathbb{1}_{i \in \mathcal{P}} \sigma_{P}+\left(1-\mathbb{1}_{i \in \mathcal{P}}\right) \sigma_{C}\right)\left(\mathbb{1}_{j \in \mathcal{C}} \sigma_{C}+\left(1-\mathbb{1}_{j \in \mathcal{C}}\right) \sigma_{P}\right){ }^{4}$

Regardless of the specific design $\mathcal{D}$, customers are directly involved during service delivery as they provide the necessary input (e.g., information about symptoms, samples for lab work, etc; see Sampson and Froehle 2006). Given this "personal" involvement, we assume that customers are risk averse in that they prefer less variable and more uniform service experiences. We utilize the mean-variance utility formulation (Markowitz 1952) to express the customer's expected utility as $\mathbb{E}[U(V(\mathcal{D}))]=\mathbb{E}[\tilde{V}]-\frac{r}{2} \operatorname{Var}[\tilde{V}]=V(\mathcal{D})-$ $\frac{r}{2} \sigma^{2}(\mathcal{D})$ where $U(\cdot)$ is a concave increasing utility function and $r>0$ is customers' measure of risk aversion (Varian 1992). Accordingly, the expected consumer surplus is given by

\footnotetext{
${ }^{4}$ The indicator function $\mathbb{1}_{i \in \mathcal{P}}=1$ if step $i$ is controlled by the provider and $\mathbb{1}_{i \in \mathcal{P}}=0$ if it is controlled by the customer. Similarly, $\mathbb{1}_{j \in \mathcal{C}}=1$ if step $j$ is controlled by the customer and $\mathbb{1}_{j \in \mathcal{C}}=0$ if it is controlled by the provider.
} 
$\mathbb{E}[U(V(\mathcal{D}), p)]=\mathbb{E}[\tilde{V}]-\frac{r}{2} \operatorname{Var}[\tilde{V}]-p=V(\mathcal{D})-\frac{r}{2} \sigma^{2}(\mathcal{D})-p$. Table 1 summarizes the notation used throughout the paper.

Table $1 \quad$ Notation, $i \in \mathcal{J}=\{1,2, \ldots, n\}$.

\begin{tabular}{lll}
\hline Parameters & Symbol & Definition \\
\hline$n$ & Total number of service steps. \\
& $V_{i}^{P}$ & Functional benefit when the provider controls step $i$. \\
& $V_{i}^{C} \lessgtr V_{i}^{P}$ & Functional benefit when the customer controls step $i$. \\
& $\sigma_{P}^{2} \doteq\left(\delta \sigma_{P}\right)^{2}$ & Variance of the experience when the provider controls a step. \\
$\delta$ & Variance of the experience when the customer controls a step. \\
$\rho$ & Relative variance. \\
$r$ & Correlation of the experiences at the different service steps. \\
& $c_{i}$ & Customer's risk aversion. \\
& Provider's marginal cost of performing step $i$. \\
\hline Decision Variables & \\
\hline & & \\
$\mathcal{D}=(\mathcal{P}, \mathcal{C})$ & Definition \\
$\mathcal{P} \subseteq \mathcal{J}$ & Service design. \\
$\mathcal{C}=\mathcal{J} \backslash \mathcal{P}$ & Set of steps controlled by the provider. \\
\hline
\end{tabular}

Without loss of generality, we normalize the price that the customers are willing to pay when $\mathcal{D}=(\emptyset, \mathcal{J})$ to zero. Hence, customers deem the service beneficial if and only if their expected surplus $\mathbb{E}[U(V(\mathcal{D}), p)]$ exceeds the surplus $\mathbb{E}[U(V(\mathcal{D}=(\emptyset, \mathcal{J})), p=0)]=$ $V((\emptyset, \mathcal{J}))-\frac{r}{2} \sigma^{2}((\emptyset, \mathcal{J}))$ which they expect to enjoy from self-performing the entire service.

\subsection{The Service Provider's Design Decisions}

We formulate the service provider's design problem under two settings: i) the benchmark setting of full information and ii) a setting of asymmetric information. In the full information case, the customers' capabilities to perform the service steps are fairly homogeneous (i.e., all customers have similar capabilities) and known to the service provider (i.e., the service provider can observe or estimate these capabilities). In the asymmetric information case, customers differ with respect to their capabilities and the provider cannot directly observe them. The service provider determines the design $\mathcal{D}=(\mathcal{P}, \mathcal{C})$. Given that $\mathcal{C}=\mathcal{J} \backslash \mathcal{P}$, by determining the optimal $\mathcal{P}$ the provider also determines the optimal $\mathcal{C}$. Hence, in the rest of the paper, we refer to $\mathcal{P}$ as the main design decision in addition to the price $p$ charged for the service. 
In the full information case, the provider serves a homogeneous (in customer capabilities) market by designing a single service. The optimal service design is based on:

$$
\begin{aligned}
\underset{\mathcal{P} \subseteq \mathcal{J}, p \geq 0}{\operatorname{maximize}} \Pi(\mathcal{P}, p)=\left(p-\sum_{i \in \mathcal{P}} c_{i} V_{i}^{P}\right) M \\
\text { s.t. } \mathbb{E}[U(\mathcal{P}, p)] \geq \mathbb{E}[U(\mathcal{P}=\emptyset, p=0)]
\end{aligned}
$$

In the provider's profit function, $M$ indicates the size of the market, which we normalize to one without loss of generality, and $c_{i} \in(0,1)$ represents the marginal cost (per unit of $V_{i}^{P}$ ) of controlling service step $i$. The total cost that the provider incurs from controlling step $i$ is $c_{i} V_{i}^{P}$, which captures the fact that steps of higher service value/quality are also more expensive to provide. For ease of exposition, we normalize to zero the cost that the provider incurs when a service step is controlled by the customer (i.e., $c_{i}=0$ for all $i \in \mathcal{C}$ ). Our cost structure aims to capture the cost savings that the provider enjoys by delegating service steps to the customers. Hence, fixed costs incurred independent of whether a service step is controlled by the provider or the customer (e.g., purchase of cooking equipment, rental of service facility) are also normalized to zero. It could be argued that such costs can be amortized over the $\operatorname{cost} c_{i} V_{i}^{P}$ that the provider incurs when controlling a step $i$.

In practice, controlling a larger part of the service process may impose additional fixed costs or capacity considerations to the provider. For instance, it may be necessary to hire additional servers who are paid over a certain time period regardless of their workload. Our model implicitly assumes a capacity scheduling flexibility and "distributes" such fixed costs over the additional value that a customer derives when the provider controls a service step. Such considerations point to operational aspects of service design, which, however, fall outside the scope of this study. We believe that a more detailed treatment of these issues in the context of allocating customer control of the service process is a promising direction for future research.

In the asymmetric information case, the provider serves a heterogeneous (in customer capabilities) market by designing a service line as opposed to a single service. We consider customers to be differentiated with respect to their average capability to perform the different service steps. The average step capability of a $\theta$-type customer is $\bar{V}^{C}(\theta)=$ $\sum_{i=1}^{n} V_{i}^{C}(\theta) / n$, where any $\theta_{1}>\theta_{0}$ implies $\bar{V}^{C}\left(\theta_{1}\right)>\bar{V}^{C}\left(\theta_{0}\right)$ and $V_{i}^{C}\left(\theta_{1}\right)>V_{i}^{C}\left(\theta_{0}\right)$ for any step $i \in \mathcal{J}$. We assume that the different customer types are uniformly distributed 
according to $\bar{V}^{C}(\theta) \sim \mathcal{U}\left[0, \bar{V}^{P}\right]$, where $\bar{V}^{P}=\sum_{i=1}^{n} V_{i}^{P} / n$. Hence, we formulate the case of information asymmetry by using the average quantities $\bar{V}^{P}, \bar{V}^{C}$, and $\bar{c}$, in the place of $V_{i}^{P}$, $V_{i}^{C}$, and $c_{i}$, respectively and we focus on the meaningful case where the average marginal benefit that the provider can derive is positive (i.e., $\left.\left(\bar{V}^{P}-\bar{V}^{C}\right)-\bar{c} \bar{V}^{P}>0\right)$.

The provider offers two services: $\mathcal{D}_{0}=\left(p_{0}, \mathcal{P}_{0}\right)$ and $\mathcal{D}_{1}=\left(p_{1}, \mathcal{P}_{1}\right)$ targeting the lower and higher end of the market, respectively. The optimal service line design is based on:

$$
\begin{aligned}
& \underset{\mathcal{P}_{1}, \mathcal{P}_{0} \subseteq \mathcal{J}, p_{1}, p_{0} \geq 0}{\operatorname{maximize}} \Pi\left(\mathcal{P}_{1}, \mathcal{P}_{0}, p_{1}, p_{0}\right)=\left(p_{0}-\sum_{i \in \mathcal{P}_{0}} \bar{c} \bar{V}^{P}\right) M_{0}+\left(p_{1}-\sum_{i \in \mathcal{P}_{1}} \bar{c} \bar{V}^{P}\right) M_{1} \\
& \text { s.t } M_{0}\left(\mathcal{P}_{0}, \mathcal{P}_{1}, p_{0}, p_{1}\right) \doteq \frac{\bar{V}^{C}\left(\hat{\theta}_{0}\right)}{\bar{V}^{P}}>0 \\
& \\
& M_{1}\left(\mathcal{P}_{0}, \mathcal{P}_{1}, p_{0}, p_{1}\right) \doteq \frac{\bar{V}^{C}\left(\hat{\theta}_{1}\right)-\bar{V}^{C}\left(\hat{\theta}_{0}\right)}{\bar{V}^{P}}>0 \\
& M_{0}\left(\mathcal{P}_{0}, \mathcal{P}_{1}, p_{0}, p_{1}\right)+M_{1}\left(\mathcal{P}_{0}, \mathcal{P}_{1}, p_{0}, p_{1}\right) \leq 1,
\end{aligned}
$$

where $\bar{V}^{C}\left(\hat{\theta}_{0}\right) \doteq\left\{\bar{V}^{C}(\theta): \mathbb{E}\left[U\left(\mathcal{P}_{0}, p_{0}\right)\right]-\mathbb{E}\left[U\left(\mathcal{P}_{1}, p_{1}\right)\right]=0\right\}$ is the average capability of the customer who is indifferent between the two services and $\bar{V}^{C}\left(\hat{\theta}_{1}\right) \doteq$ $\left\{\bar{V}^{C}(\theta): \mathbb{E}\left[U\left(\mathcal{P}_{1}, p_{1}\right)\right]-\mathbb{E}[U(\mathcal{P}=\emptyset, p=0)]=0\right\}$ is the average capability of the customer who is indifferent between receiving the service $\left(p_{1}, \mathcal{P}_{1}\right)$ from the provider and selfperforming it.

By determining the optimal prices $p_{0}, p_{1}$, and sets of steps $\mathcal{P}_{0}, \mathcal{P}_{1}$, the provider determines the optimal market segmentation (i.e., the number of customers $M_{0}$ and $M_{1}$ to receive each service). Our formulation can extend to service lines comprising multiple (i.e., more than two) service offerings. We focus on two offerings, which allows us to capture the essence of the design trade-offs that the provider faces when serving a heterogeneous customer base and at the same time to maintain expositional parsimony.

\section{Analysis and Results}

In this section, we analyze the service provider's optimal design decisions. In $\S 4.1$ we focus on the case of full information where customers' capabilities to perform the service steps are homogeneous and known to the provider. In $\S 4.2$ we extend our analysis to the case of asymmetric information where customers' capabilities are heterogeneous and unknown to the service provider. All proofs and analytical expressions are relegated to the Appendix. 


\subsection{Service Design under Full Information}

The service provider determines the set of steps $\mathcal{P}$ for which she assumes responsibility and the price $p$. With respect to pricing, for any $\mathcal{P}$, the service provider chooses the maximum price $\bar{p}(\mathcal{P})$ (see Appendix) for which customers deem the service beneficial.

With respect to the design configuration, let $\mathcal{F}$ indicate the power set of $\mathcal{J}$. There are $2^{n}$ elements in $\mathcal{F}$ among which the provider chooses the $\mathcal{P}^{*} \subseteq \mathcal{F}$ that maximizes her profit. The fact that the service steps are heterogeneous (i.e., they have different $V_{i}^{P}, V_{i}^{C}$, and/or $\left.c_{i}\right)$, implies that each step's contribution to the provider's profit differs. We define $A_{i} \doteq$ $\left(V_{i}^{P}-V_{i}^{C}\right)-c_{i} V_{i}^{P}$ as the expertise-based marginal benefit that the provider derives when she controls step $i \in \mathcal{J}$. The first term, $V_{i}^{P}-V_{i}^{C}$, of $A_{i}$ captures the marginal functional value added due to the provider's expertise advantage whereas, the second term, $c_{i} V_{i}^{P}$, captures the marginal cost incurred from controlling step $i$. Our first proposition describes the priority rule the provider follows to determine which steps to control. We use $k \in[0, n]$ to denote the cardinality of $\mathcal{P}$.

Lemma 1. (Optimal Design: The Choice Rule) To determine which service steps to control, the provider sorts all steps in the decreasing order of their marginal benefit $A_{i}$, $\forall i \in \mathcal{J}$. Let $A_{[j]}$ indicate the $j^{\text {th }}$ element of this ordering. The optimal partitioning of the service steps is $\mathcal{P}=\left\{[1],[2], \ldots,[j]: j=k^{*}\right\}$ and $\mathcal{C}=\mathcal{J} \backslash \mathcal{P}$, where $k^{*} \in[0, n]$.

Lemma 1 outlines a practical policy, which is based on an intuitive economic foundation: the marginal benefit that the service provider can derive from each step. The provider prioritizes the steps that offer higher marginal benefit. This benefit is determined by the extent to which the provider's expertise advantage $V_{i}^{P}-V_{i}^{C}$ at a service step outweighs the $\operatorname{cost} c_{i} V_{i}^{P}$ of the step. Hence, this choice policy reflects the joint effect of $V_{i}^{P}, V_{i}^{C}$, and $c_{i}$ on the provider's decision regarding which steps to control. ${ }^{5}$

Although Lemma 1 outlines how the provider should prioritize which steps to control, it does not characterize the actual design, that is, how many steps of this priority ordering she optimally selects. To this end, the next finding determines the cardinality $k^{*}$ of $\mathcal{P}^{*}$. For tractability, we approximate $k \in[0, n]$ to be a continuous variable ${ }^{6}$ and we assume that

\footnotetext{
${ }^{5}$ In its most general form $A_{i} \doteq\left(\left(V_{i}^{P}-V_{i}^{C}\right)-c_{i} V_{i}^{P}\right) M-F_{i}$, our choice rule can capture cases where controlling a step $i$ imposes an incremental fixed cost $F_{i}$ to the provider.

${ }^{6}$ In the Appendix, we show that the provider's profit is "well-behaved" (i.e., continuous and concave) in $k$. Hence, the optimal discrete number of steps can be determined by approximating $k^{*} \in[0, n]$ to the closest integer $\left\lceil k^{*}\right\rceil$ or $\left\lfloor k^{*}\right\rfloor$ that results in higher profit.
} 
$A(k)=A_{[1]}-\frac{A_{[1]}-A_{[n]}}{n} k$, where $A_{[1]}$ and $A_{[n]}$ is the maximum and minimum value of $A_{i}$, respectively. To capture a wide range of services, in the rest of the paper we assume that $A_{[1]}>0$ and $A_{[n]} \leq 0$ regardless of the service context. In other words, we consider settings where the provider does not have an a priori advantage for all possible steps.

Lemma 2. (Optimal Design: The Number of Steps) The optimal number of steps controlled by the provider is $k^{*}=\max \left\{\min \left\{\frac{n\left(2 A_{[1]}-(1-\delta) r \sigma_{P}^{2}(1-\rho+\delta+(2 n-1) \delta \rho)\right)}{2\left(A_{[1]}-A_{[n]}+(1-\delta)^{2} n \rho r \sigma_{P}^{2}\right)}, n\right\}, 0\right\}$. All else being equal, a service provider controls at least as many steps in routine as in nonroutine services.

Lemma 2 draws a significant managerial implication for service designs. Ceteris paribus, services that are primarily conformance quality-orientated (i.e., routine services) require more control from the provider than non-routine services. Said differently, designers should think of routine services as "blocks" of steps that need to be managed holistically. Instead, non-routine services should be focused on the "core provision." The reason is that in routine services more control always benefits the customers because it results in greater cumulative functional value and more consistent experience. In non-routine services, the same level of provider involvement possibly increases the overall variability of the customer experience and limits the eventual value to the customer.

We should note that our model does not assume a relationship between expertise and variability advantage. In practice, there may be service settings where the provider's expertise is a proxy for her ability to manage variability. For instance, a provider of high expertise may be able to offer a consistent service all the time. In such cases, the provider optimally controls a larger number of steps $k^{*}$ under both routine and non-routine services.

We have also assumed that customers prefer less variable and more consistent service experiences. In cases where customers exhibit high tolerance or even favor experiential variability (e.g., they prefer to have different experiences every time they visit a service facility) the provider would control a larger number of steps under non-routine and a smaller number under routine services. Our analysis has deliberately stayed away from confounding effects between the functional and idiosyncratic parts of the customer value in order to delineate them in a clean fashion. 
Corollary 1. (Optimal Design: Holistic vs. Focused Control) In non-routine services, the provider may choose to delegate steps for which she is more capable of performing than the customer and at which she would derive a positive marginal benefit. In routine services, the provider may control steps for which she is less capable of performing than the customers and at which she derives a negative marginal benefit.

Through the control of a step in a non-routine service, the provider contributes to the customer value with the added functional value she provides. But at the same time, she also increases the variability of the overall customer experience. The provider balances this trade-off by optimally controlling steps for which she maintains a sufficient expertise advantage over the customers. This does not imply that the provider controls all steps for which $V_{i}^{P}>V_{i}^{C}$. Rather, the provider controls only $k^{*}$ of the steps with positive marginal benefit $A(k)>0$ (see Figure 2a). In contrast, in routine services, the provider always finds it optimal to control more steps than simply the ones with $A(k)>0$, hence, steps with a negative marginal benefit $A(k)<0$ (see Figure $2 \mathrm{~b}$ ). This implies that the provider controls steps where she holds lower expertise than the customers (i.e., $V_{i}^{P}<V_{i}^{C}$ ). Yet, from the customer's point of view, such expertise disadvantage is offset by the reliability benefit ensured due to the provider's control.

Figure 2 Optimal number of steps controlled with respect to the marginal benefit derived by the provider.

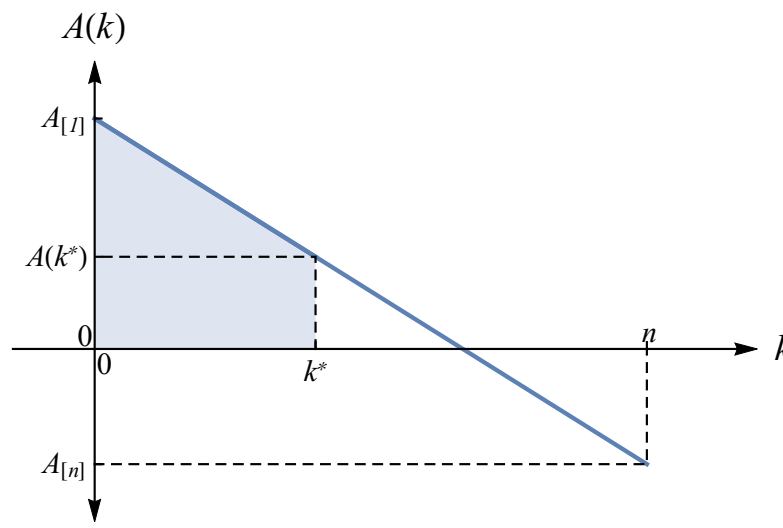

(a) Non-routine services $(\Delta \sigma>0)$

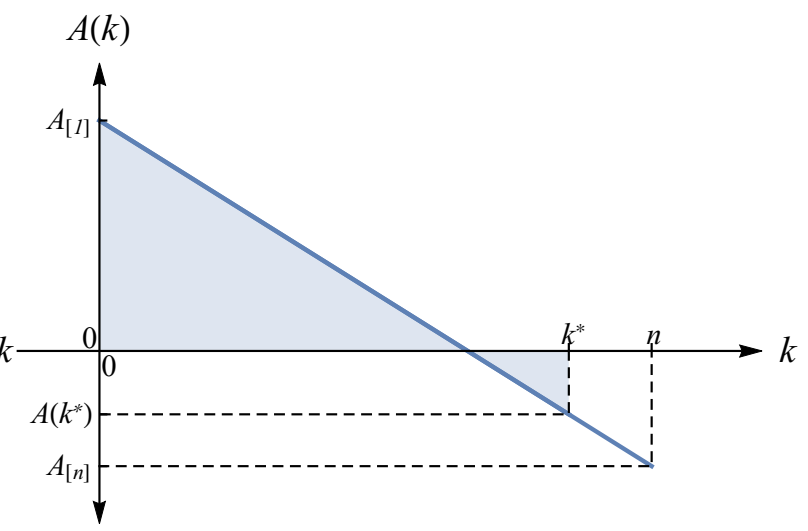

(b) Routine services $(\Delta \sigma<0)$

Note. $A_{[1]}$ and $A_{[n]}$ denote the maximum and minimum value of the marginal benefit $A_{i} \doteq\left(V_{i}^{P}-V_{i}^{C}\right)-c_{i} V_{i}^{P}$, respectively. The area $\int_{0}^{k^{*}} A(k) d k$ represents the total benefit the provider derives when she controls $k^{*} \in[0, n]$ steps.

In the next finding, we describe how the different characteristics of the service process affect the provider's decision regarding how many service steps to control. In particular, 
we consider the effect of process length $n$ and the interdependency $\rho$ of the different service steps. We focus on the cases with $k^{*} \in(0, n)$, as in extreme design solutions (i.e., $k^{*}=0$ or $k^{*}=n$ ) such local perturbations leave the design mostly the same and bear little insight.

Proposition 1. (Process Characteristics: Length and Step InterdependenCIES) For non-routine services: $\partial k^{*} / \partial n>0$ iff $n<\hat{n}$, and $\partial k^{*} / \partial \rho<0$ iff $\sigma_{P}^{2}<\hat{\sigma}_{P_{1}}^{2}$. For routine services: $\partial k^{*} / \partial n>0$, and $\partial k^{*} / \partial \rho>0$ iff $\sigma_{P}^{2}<\hat{\sigma}_{P_{2}}^{2}$.

Proposition 1 prescribes that in routine services, the provider controls a bigger part of services that comprise a larger number of steps (larger $n$; see Figure $3 \mathrm{~b}$ ), or processes with more interdependent steps (larger $\rho$ ). The reason is that all else being equal, the length and interdependencies of the service process amplify the variability of the overall customer experience. Thus, the provider increases her control over the process steps to extract more consumer surplus, as the customers benefit more from her ability to deliver the service reliably. When considering the interdependencies of the steps though, beyond a level of variability the provider does not increase $k^{*}$ because the levels of variability render the option of self-performing the service less appealing to the customers. Hence, the provider can limit her control to enjoy the related cost savings, and still have the customers deem the service beneficial.

In contrast to routine services, in non-routine services the provider increases her control only when the length (captured by the number of steps) of the process is contained (see Figure 3a). Otherwise, the added functional value fails to outweigh the losses in customer utility due to the higher overall variability. Furthermore, the provider assumes limited control in processes with highly interdependent tasks. Larger $\rho$ implies that the adverse effect of variability becomes more pronounced as negative experiences at different steps can cascade more impactfully throughout the entire service process. Hence, controlling more steps implies an exponentially larger number of interdependencies. The provider mitigates this effect by limiting $k^{*}$. Once more, the result illustrated in Figure 3a resonates with the overarching message of our analysis: the design of non-routine services must carefully focus on a subset of high-marginal benefit steps.

Next, we characterize the effect of process heterogeneity on the optimal service design. A service process is heterogeneous when the functional value is primarily offered through a small number of steps and homogeneous when all steps contribute similar functional 
Figure 3 Optimal number of steps $k^{*}$ with respect to the total number of steps $n$ of the service.

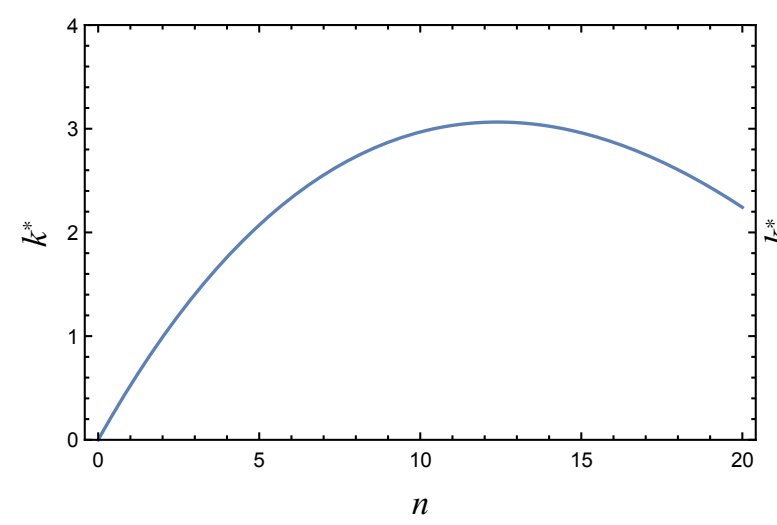

(a) Non-routine services $(\Delta \sigma>0$ with $\delta=0.5)$

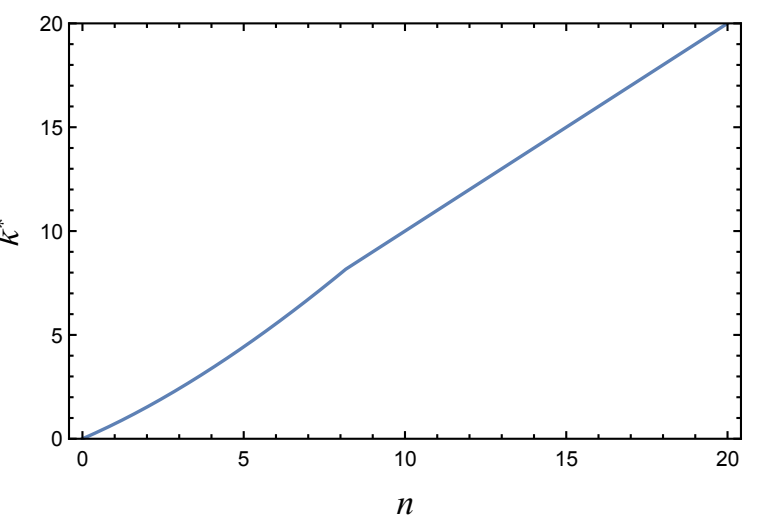

(b) Routine services $(\Delta \sigma<0$ with $\delta=1.5)$

Note. We calculate $k^{*}$ using $r=0.45, A_{[1]}=2.25, A_{[n]}=-1.5, \rho=0.4$, and $\sigma_{P}^{2}=1.66$.

value. We address this comparison to understand how the distribution of value within a service process plays any role in shaping the optimal design. In this way, we can answer the question of whether designs should differ when the majority of the value comes from a few core service steps. In order to make the comparison "unbiased," we restrict the total benefit that the provider can derive by controlling the entire service to be the same and equal to $\frac{A_{[1]}+A_{[n]}}{2} n$, under both a homogeneous and a heterogeneous process. Their difference is that for the heterogeneous service process $\mathcal{J}$ we consider $A(k)=A_{[1]}-\frac{A_{[1]}-A_{[n]}}{n} k$, whereas for the equivalent homogeneous process $|\mathcal{J}|$ we consider $A(k)=\frac{A_{[1]}+A_{[n]}}{2}$ for all $k \in[0, n]$ (see Figure 4a). We derive and compare the optimal number of steps $k^{*}(\mathcal{J})$ and $k^{*}(|\mathcal{J}|)$ that the provider controls in a heterogeneous and homogeneous service process, respectively along with the resulting profits $\Pi^{*}(\mathcal{J})$ and $\Pi^{*}(|\mathcal{J}|)$.

Proposition 2. (Process Characteristics: Step Heterogeneity) In nonroutine services, the provider controls $k^{*}(\mathcal{J})<k^{*}(|\mathcal{J}|)$ iff $A_{[1]}>\hat{A}_{[1]}$. In routine services, the provider controls $k^{*}(\mathcal{J})<k^{*}(|\mathcal{J}|)$ iff $A_{[1]}>\tilde{A}_{[1]}$, where $\tilde{A}_{[1]}<\hat{A}_{[1]}$. Regardless of the type of the service, the provider always derives larger profit under a heterogeneous process, i.e., $\Pi^{*}(\mathcal{J})>\Pi^{*}(|\mathcal{J}|)$.

For the same number of steps $k \in(0, n)$, a homogeneous process limits the total benefit that the provider can derive (see Figure $4 \mathrm{~b}$ ). This is because in a heterogeneous process the provider can prioritize her control and choose the more beneficial steps. For that reason, the provider's profit is always higher under a heterogeneous than a homogeneous process. 
Figure 4 Heterogeneous versus homogeneous service process.

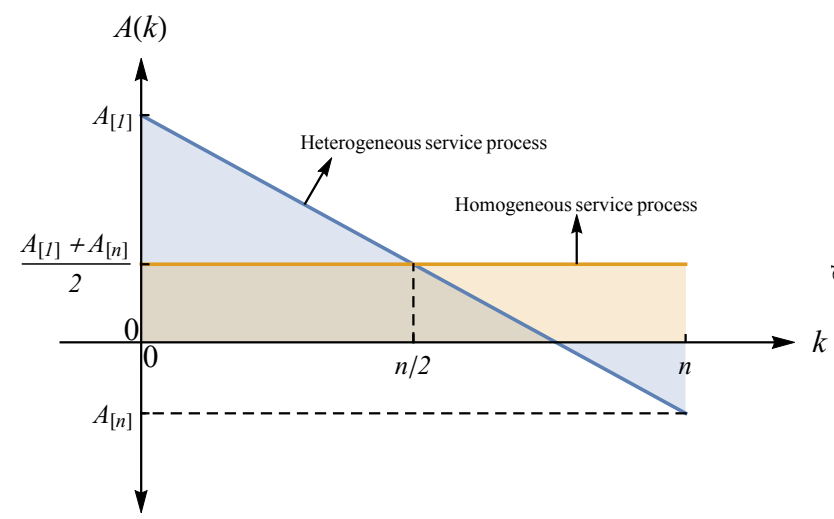

(a) Marginal benefit $A(k)$

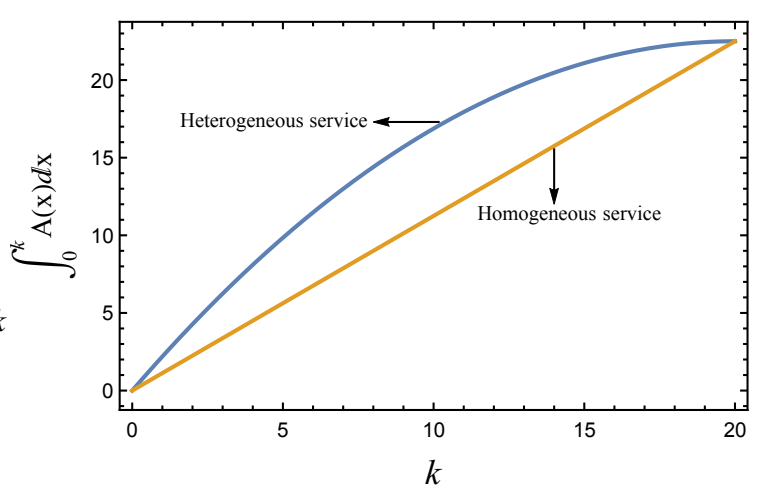

(b) Total benefit $\int_{0}^{k} A(x) d x$

Note. The total benefit that the provider can extract $\int_{0}^{n} A(k) d k$ is the same for both service processes. The total benefit $\int_{0}^{k} A(x) d x$ is calculated using $n=20, A_{[1]}=2.25$, and $A_{[n]}=0$.

Although the provider's profit is always higher under a heterogeneous service process, the extent of the control under either process depends on the extent of her maximum expertise advantage. We find that for larger values of expertise advantage, the provider exercises more control in homogeneous processes. Interestingly, for smaller levels of expertise advantage, the provider controls more steps when the service process is heterogeneous.

A heterogeneous distribution of functional value across the steps would naturally push the service provider to focus on the fewer steps where she holds the largest expertise advantage. Yet, for the same $k \in(0, n)$, a homogeneous process bounds from both above and below the marginal benefit that the provider can extract. Hence, the provider offers the service only if it comprises a sufficient number of steps for which she maintains an expertise advantage over the customers. The larger the number of such potential steps, the higher the number of steps the provider controls. After a certain point, the incremental benefit of controlling an additional step is larger in a homogeneous than in a heterogeneous process. For that reason, for smaller $A_{[1]}$ the provider controls more steps in a heterogeneous process. The counterintuitive nature of this result points out our earlier call for understanding the relative capability of the provider and the customer in order to carefully assess the marginal benefit of the service steps.

The above result holds true regardless of whether the service is routine or non-routine (see Figure 5). The only difference is that in routine services, the range of $A_{[1]}$ values where the provider exercises more control in heterogeneous processes is smaller. Hence, in routine services, the provider tends to control more steps in homogeneous processes. 
Figure 5 The optimal number of steps $k^{*}$ controlled by the provider for homogeneous and heterogeneous service processes.

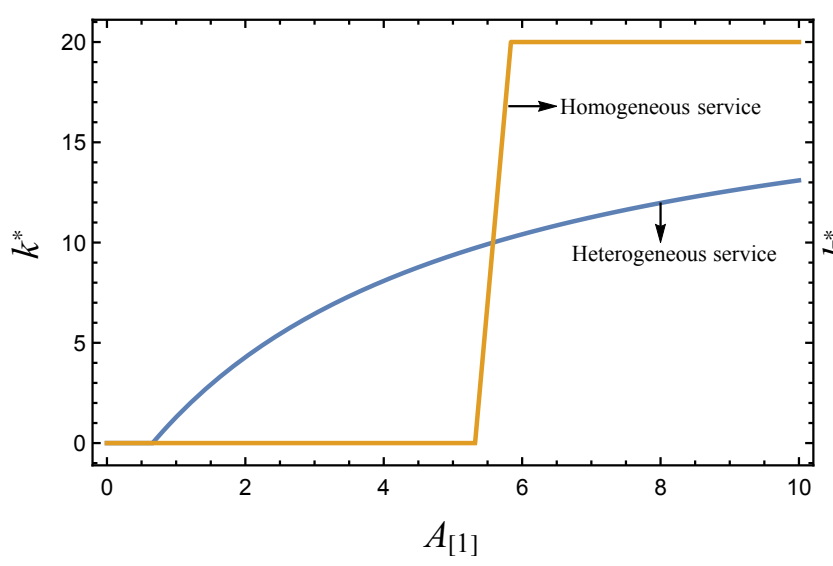

(a) Non-routine services $(\Delta \sigma>0$ with $\delta=0.7)$

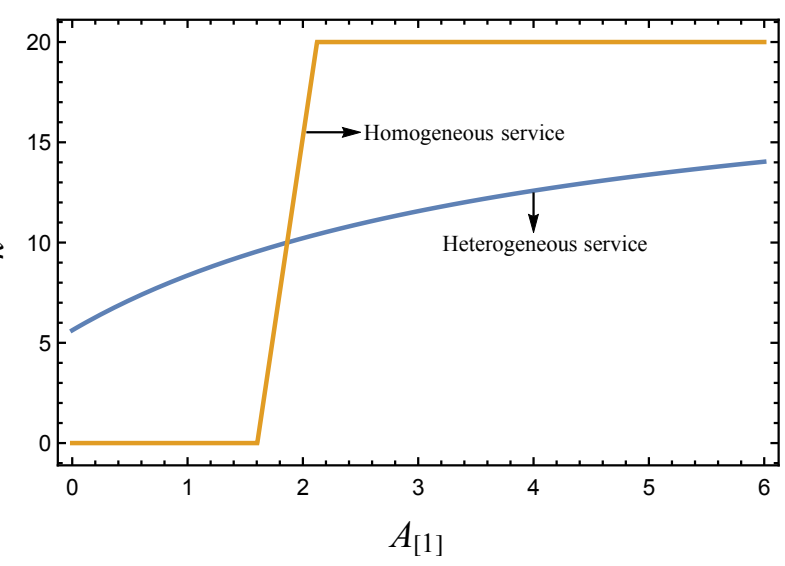

(b) Routine services $(\Delta \sigma<0$ with $\delta=1.3)$

Note. We calculate $k^{*}$ using $n=20, r=0.45, A_{[n]}=-4, \rho=0.4$, and $\sigma_{P}^{2}=0.8$.

\subsection{Service Design under Information Asymmetry}

In this section, we extend our analysis to the case of asymmetric information: customers' capabilities are heterogeneous and unknown to the service provider. As we discussed in $\S 3$, customers are differentiated with respect to their average capability to perform the different service steps. Our next finding describes the effect of such heterogeneity in customer capability on the provider's profitability. In particular, we compare the provider's optimal profit under a heterogeneous market where the different types of customers $\theta$ are distributed according to $\bar{V}^{C}(\theta) \sim \mathcal{U}\left[0, \bar{V}^{P}\right]$, with the optimal profit under a homogeneous market where all customers have the same type $|\theta|$ and $\bar{V}^{C}(|\theta|)=\bar{V}^{P} / 2$. Thus, the customers of the homogeneous market have the same capability as the "average" customer of the heterogeneous market.

Proposition 3. (The Effect of Customer Heterogeneity) In non-routine services, customer heterogeneity results in higher (lower) profit for larger (smaller) values of experiential variability, i.e., $\Pi^{*}(\theta)-\Pi^{*}(|\theta|)>0$ iff $\sigma_{P}^{2}>\bar{\sigma}_{P}^{2}$. In routine services, customer heterogeneity results in higher (lower) profit for smaller (larger) values of variability, i.e., $\Pi^{*}(\theta)-\Pi^{*}(|\theta|)>0$ iff $\sigma_{P}^{2}<\overline{\bar{\sigma}}_{P}^{2}$.

Customer heterogeneity implies an information asymmetry since the provider cannot know (and it would be too costly to infer) the exact "type" (i.e., capability) of each customer in the market. This means that, unlike the case of a homogeneous market, when 
the customers are heterogeneous, the provider fails to extract the entire consumer surplus through a single service offering. Hence, it is reasonable to expect customer heterogeneity to affect the provider's profit negatively.

Proposition 3 shows that this is not necessarily true. Customer heterogeneity moderates the negative effect of variability on the provider's profitability. In non-routine services, the provider's profit decreases in $\sigma_{P}^{2}$ regardless of whether the market is homogeneous or heterogeneous (see Figure 6a). This is because in the face of larger variability, risk averse customers opt out of undertaking the service and as a result, the provider decreases the price she charges (see Figure 6c) or limits her control, which reduces her profit. This decrease, however, is less detrimental when the market is heterogeneous as the provider is able to focus on a smaller and less capable part of the market (i.e., she is able to segment the market), which affords her to retain her pricing or even charge a higher price. Although this segmentation allows for a pricing advantage, and as an extension, margin advantage, it limits the number of customers receiving the service creating a volume disadvantage. For that reason, when the variability is smaller, the provider derives higher profit in a homogeneous than a heterogeneous market (see Figure 6a) as she can charge a high price and still attract a large number of customers (see Figure $6 \mathrm{~b}$ ).

Figure 6 Optimal profit $\Pi^{*}$, price $p^{*}$ and market coverage $M^{*}$ of non-routine services under a heterogeneous $\left(\bar{V}^{C}(\theta) \sim \mathcal{U}\left[0, \bar{V}^{P}\right]\right)$ versus a homogeneous $\left(\bar{V}^{C}(|\theta|)=\bar{V}^{P} / 2\right)$ market.

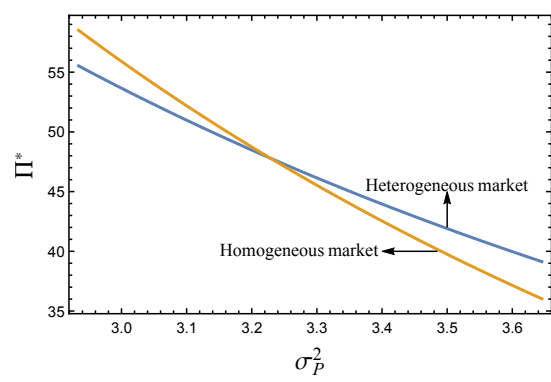

(a) Profit

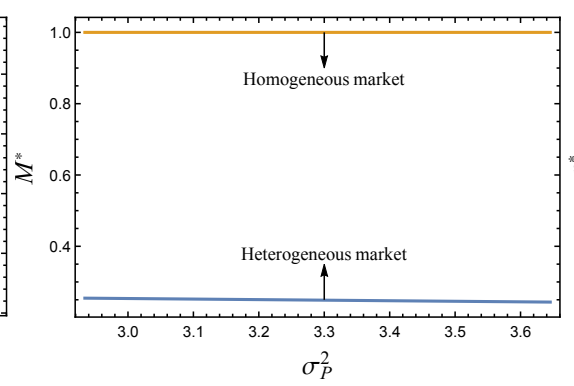

(b) Market coverage

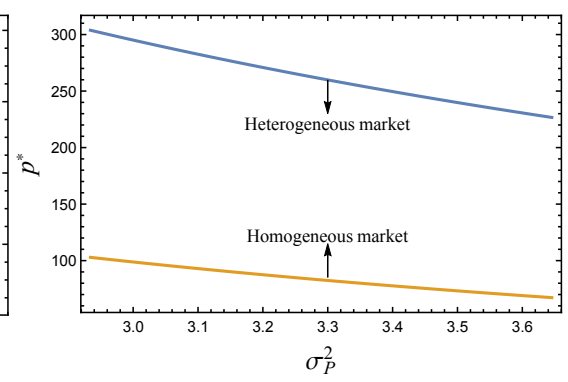

(c) Price

Note. We calculate $\Pi^{*}, p^{*}$, and $M^{*}$ using $\delta=0.75, n=7, r=0.75, \bar{V}^{P}=10, \bar{c}=0.1$ and $\rho=0.3$.

In routine services, we observe the opposite behavior. The fact that the provider can perform the service more reliably than the customers implies that larger values of $\sigma_{P}^{2}$ increase the provider's variability advantage (we remind the reader that in routine services $\delta>1$ and $\left.\Delta \sigma \doteq \sigma_{P}-\sigma_{C}=(1-\delta) \sigma_{P}<0\right)$. This allows her to charge higher prices (see Figure 7c) 
and still attract enough customers regardless of whether they have the same or different capabilities. In the case of a heterogeneous market, larger values of $\sigma_{P}^{2}$ enable the provider to also increase her market coverage (see Figure 7b). Doing so implies that the provider reaches out to the higher end of the market (i.e., to the high-capability customers). This, however, limits the extent to which she can increase the price she charges. All else being equal, a more capable customer is willing to pay a smaller price than a less capable customer to receive the service from the provider. Hence, for larger values of variability the provider benefits more from offering a routine service in a homogeneous than in a heterogeneous market. The reverse is true as the provider's variability advantage decreases (i.e., for smaller values of $\sigma_{P}^{2}$ ). In this case, the provider derives higher profit in a heterogeneous market (see Figure 7a) because she can focus on the less capable customers.

Figure 7 Optimal profit $\Pi^{*}$, price $p^{*}$ and market coverage $M^{*}$ of routine services under a heterogeneous $\left(\bar{V}^{C}(\theta) \sim\right.$ $\left.\mathcal{U}\left[0, \bar{V}^{P}\right]\right)$ versus a homogeneous $\left(\bar{V}^{C}(|\theta|)=\bar{V}^{P} / 2\right)$ market.

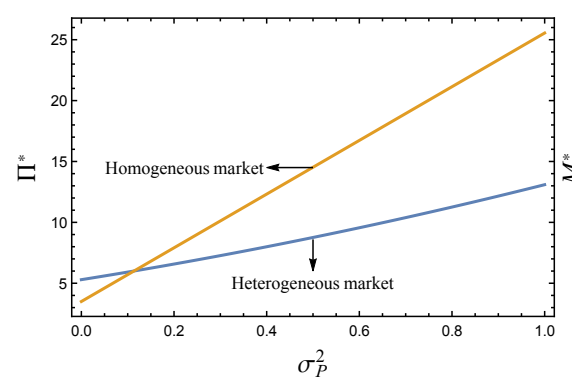

(a) Profit

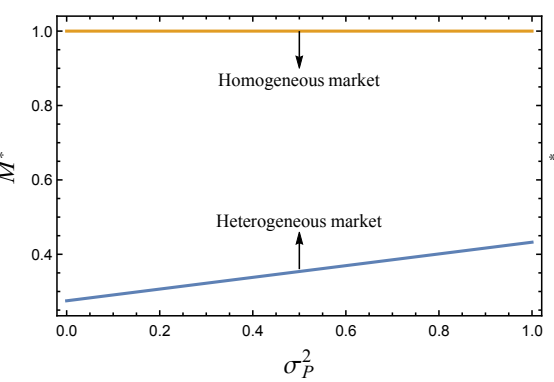

(b) Market coverage

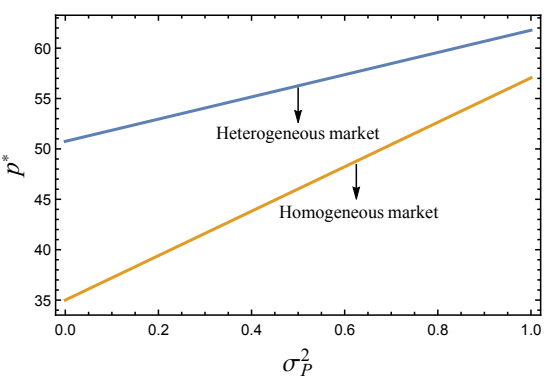

(c) Price

Note. We calculate $\Pi^{*}, p^{*}$, and $M^{*}$ using $\delta=2, n=7, r=0.75, \bar{V}^{P}=10, \bar{c}=0.1$ and $\rho=0.3$.

Service Line Design. Varying capability levels among the customers may present an opportunity for the provider to offer multiple services that are better tailored to the needs of the different customers. In what follows, we characterize the economic benefit of providing multiple services. We do so by comparing the optimal profit $\Pi_{\text {line }}^{*}(\theta)$ of a service line that comprises two different services with the optimal profit $\Pi^{*}(\theta)$ of a single service.

Proposition 4. (The Economic Benefit of Service Lines) When customers are heterogeneous in their capabilities, $\Delta \Pi=\Pi_{\text {line }}^{*}(\theta)-\Pi^{*}(\theta)>0$. The economic benefit $\Delta \Pi$ exhibits an inverted " $U$-shaped" relationship with respect to the length $n$ of the service process. In non-routine services, $\partial \Delta \Pi / \partial n>0$ iff $n \in(0, \bar{n})$ and in routine services $\partial \Delta \Pi / \partial n>$ 0 iff $n \in(0, \overline{\bar{n}})$. All else being equal, the process length at which the benefit of offering a service line is maximized, is larger in non-routine than in routine services, i.e., $\bar{n}>\overline{\bar{n}}$. 
Intuitively, Proposition 4 suggests that in the presence of customer heterogeneity, the provider always derives higher profit by offering a service line. This is because a service line facilitates better market segmentation and enables the provider to extract higher consumer surplus. Proposition 4 however, points to the non-monotonic relationship between the economic benefit of offering a service line and the length of the service process. We find that a service line is more profitable for intermediate lengths. This is true for both routine and non-routine services (see Figure 8). In non-routine services, we have already discussed in Proposition 1 that for smaller or larger values of $n$, the provider optimally limits the control of the service (ultimately $k^{*} \rightarrow 0$; see Figure $3 \mathrm{a}$ ). This also extends to the case of service lines. On the contrary, in routine services, we have seen that for larger values of $n$, the provider optimally controls larger part of the service (see Figure 3b), which ultimately leads to controlling the entire service process (i.e., $k^{*} \rightarrow n$ ). This implies that for a larger number of steps, the extent to which the provider differentiates the designs of the two services decreases because she is bounded by the total number of steps $n$ that she can control. It also explains why in routine services, the benefit of offering a service line is more pronounced at smaller values of $n$.

Figure 8 The economic benefit $\Delta \Pi^{*}$ of offering a service line (i.e., two services) versus a single service in a heterogeneous market.

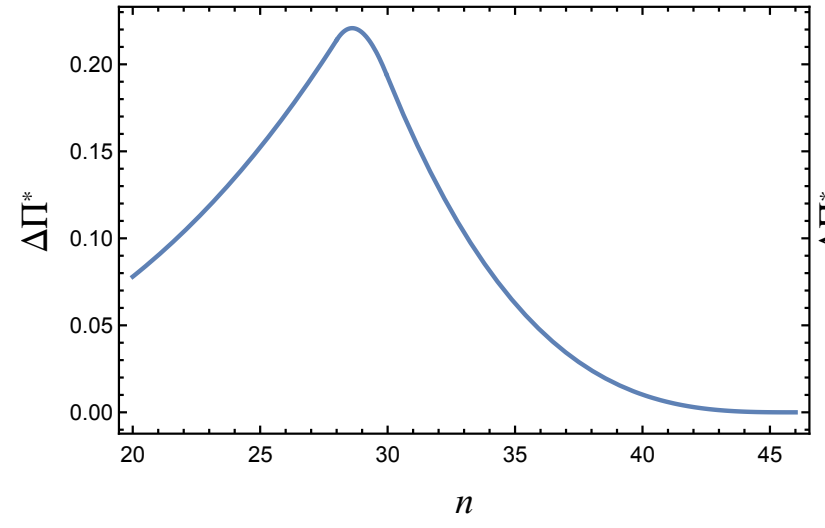

(a) Non-routine services $(\Delta \sigma>0$ with $\delta=0.7)$

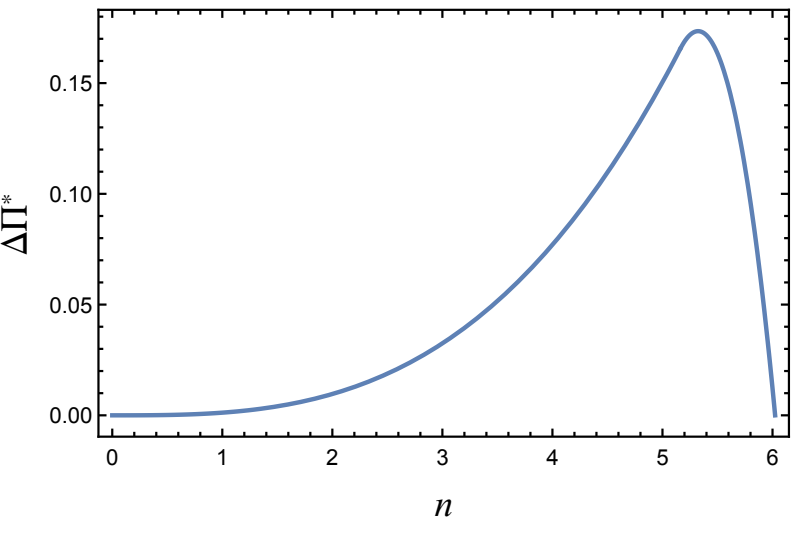

(b) Routine services $(\Delta \sigma<0$ with $\delta=2)$

Note. We calculate $\Delta \Pi^{*}=\Pi^{*}($ service line $)-\Pi^{*}($ single service $)$ using $\sigma_{P}^{2}=1.5, r=0.75, \bar{V}^{P}=10, \bar{c}=0.1$ and $\rho=0.3$.

The non-monotonic behavior captured in Proposition 4 implies that the decision to offer a line of services might be more involved. This is especially true in the case when the design and deployment of a service line entail additional costs. We elaborate on Corollary 2. 
Corollary 2. (The Economic Benefit of Service Lines) In the presence of deployment costs, offering a service line may be economically justified only for service processes of certain length.

Research on service management (Zeithaml et al. 1985) has identified one of the main differences between products and services to be the fact that services are simultaneously produced and consumed. For that reason, they cannot be inventoried. From a practical point of view, this implies that although the different variants of a line of products can be manufactured and subsequently sold by using the same pool of resources (e.g., the same production facility), this may not be true with service lines. That is, the provider may not be able to enjoy economies of scope (e.g., see Krishnan and Zhu 2006) because a service line may entail deployment costs (e.g., acquiring new facilities/equipment, hiring and training of personnel, monitoring costs), which can dampen the benefit (e.g., better segmentation) of offering more than one service. Hence, depending on the magnitude of these costs, the provider may prefer to design a service line only when the benefit (over serving the market through a single service) of doing so is maximum. As we have discussed in Proposition 4, this happens at intermediate values of process length (see Figure 8).

With this finding, our paper complements existing research on product line design (see Krishnan and Gupta 2001, Krishnan and Zhu 2006, Lacourbe et al. 2009 and references therein) by extending the focus of design to service lines. We do so by accounting for factors that are typically not present or considered to be design levers at any stage of the new product development process. For instance, products are typically designed around tangible specifications and features and do not require significant customer involvement during their production or delivery. On the contrary, services are intangible, they unfold over multiple interdependent service steps, and the decision to involve (or not) the customer at each of these steps can affect the overall customer experience.

The Effect of Competition on Service Design. Customer heterogeneity presents opportunities to the service provider to offer multiple services in order to cover customer needs better. In practice, this may also happen through different competing services. That is, the presence of heterogeneity in customers' capabilities may allow for the existence of multiple services offered by competing providers. In what follows we examine how competition affects service design. We consider two providers, $H$ and $L$, who differ along their capability $\bar{V}^{P}$, where $\bar{V}_{H}^{P}>\bar{V}_{L}^{P}$. We assume that the customers' capabilities are uniformly 
distributed according to $\bar{V}^{C}(\theta) \sim \mathcal{U}\left[0, \bar{V}_{H}^{P}\right]$. Although the modeling setup is structurally similar to the one we used to capture the case of a single provider offering two services, the fact that the two competing providers simultaneously design their services presents analytical challenges in the derivation of the optimal designs. In the Appendix, we provide the optimality conditions that determine the competing designs. Subsequently, we resort to numerical methods that allow us to fully characterize the resulting designs. We summarize our insights below.

Observation 1. (Service Design Under Competition) In the presence of competition, the high-capability provider serves the more capable customers whereas the lowcapability provider serves the less capable customers. The low-capability provider controls more steps under a competitive than a monopolistic setting. On the contrary, the highcapability provider decreases her control under a competitive setting and assumes responsibility for fewer service steps than the low-capability provider.

We find that for both routine and non-routine services, providers with competing offerings can coexist in a heterogeneous market. However, competition seems to force a clearer separation between the service designs adopted by the competing providers. The providers set their designs with opposite goals: i) a higher-end "focused service" where the provider's control of the service process is minimal and ii) a lower-end "super-service" where the provider controls the larger subset of the steps. In Figure 9 we see that for both routine and non-routine services, it is the high-capability provider who optimally chooses to limit her control and offer a focused service to the more capable customers. The less capable customers are "efficiently" served by the low-capability provider who can compensate for her lower expertise by controlling more service steps. For the same number of steps, the high-capability provider would incur a higher cost than the low-capability provider. For that reason, she chooses to serve the high-capability customers who are naturally capable to perform a larger part of the service themselves. Although the high-capability provider exercises minimal control, she extracts enough consumer surplus through the larger functional value she provides due to her higher expertise. This ordering also holds true for routine services. The low-capability provider does not have an incentive to cover the entire market because she can charge higher prices when serving the less capable customers. She does so by controlling the entire service as she maintains both an expertise and a 
variability advantage over the customers. Hence, the high-capability provider serves the remaining (i.e., higher-end) part of the market. However, to differentiate from the lowcapability provider and to limit her cost, she controls only a part of the service process. These insights reveal the effects of competition on service design that are not discerned by our previous findings.

Figure 9 Optimal number of steps $k_{H}^{*}$ and $k_{L}^{*}$ controlled under competition by a high- and low-capability provider, respectively.

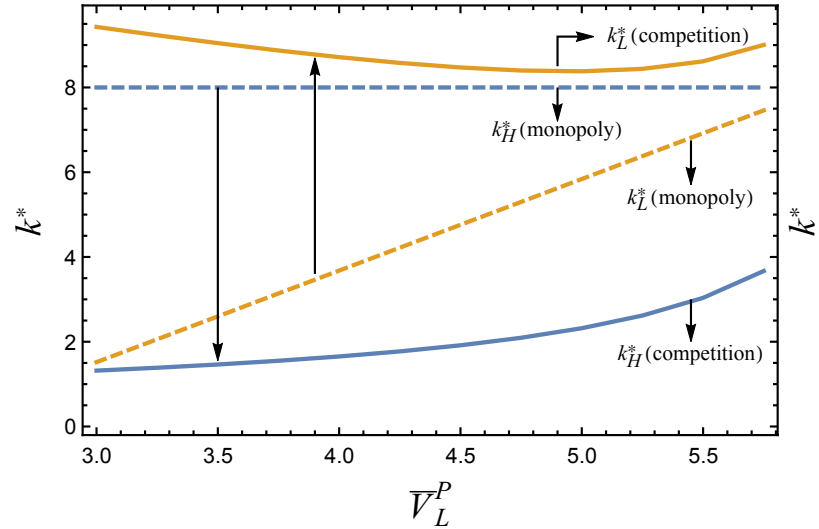

(a) Non-routine services $(\Delta \sigma>0$ with $\delta=0.4)$

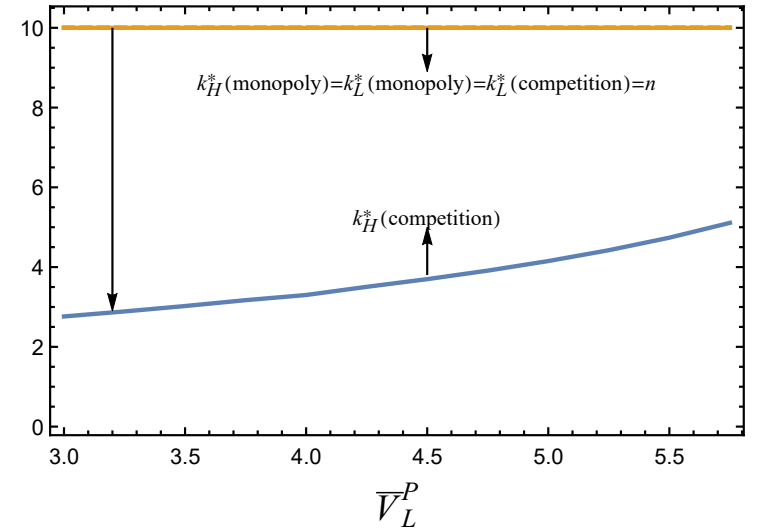

(b) Routine services $(\Delta \sigma<0$ with $\delta=1.4)$

Note. For the high-capability provider we use $\bar{V}_{H}^{P}=6$ whereas, for the low-capability provider we use $\bar{V}_{L}^{P}=[3,5.75]$. We also use $n=10, r=1, \sigma_{P}^{2}=1, \bar{c}=0.3$, and $\rho=0.6$.

\section{Discussion}

Customer participation in service contexts has been traditionally seen through the lens of the operational cost savings it can entail. Through a variety of examples, however, we recognize innovative providers who determine whether a service task should be controlled by the customer or the service firm, as a means of shaping the overall customer experience.

Motivated by these service practices, we develop a stylized analytical framework to answer the following question: under what conditions should providers grant control of different parts of their service blueprint to the customers? We consider services as processes comprising multiple interdependent steps. The experiences that customers derive at the different steps can be coupled. We distinguish between routine services where the customer appreciation of the service outcome stems from the adherence to standardized specifications, and non-routine services where the service outcome is evaluated along more subjective dimensions by the customer. Finally, we account for the possibility that the 
provider might design a service line. Our analysis generates the following insights not identified by previous research.

We characterize a step choice rule that bears a clear, intuitive economic foundation. Moreover, it lends itself to a guiding principle that providers and service designers can use to determine which service steps to control. According to this rule, managers should assign a higher priority to service steps that offer a higher marginal benefit. This benefit is determined by the relationship between the provider's expertise advantage at a service step and the total cost of controlling the step. Characterizing this relationship rests upon the thorough diligence of service designers to capture and comprehend how much more advantageous is a provider compared to the customer in performing service tasks. Methods like empathic design (Leonard and Rayport 1997) and design thinking (Brown 2008) can offer inroads towards such know-how.

The extent of the provider's control, however, differs significantly depending on the type of the service. For instance, we find that ceteris paribus the provider exercises greater control in routine services than in non-routine ones. In routine services, the provider controls steps from which she derives even a negative marginal benefit whereas, in non-routine services, she might forego steps with positive marginal benefit. This implies that routine services should be managed holistically as "blocks" of steps and that in non-routine services providers should focus on the provision of steps that manifest their core expertise. In routine services, lengthier service blueprints incentivize the provider to increase the number of steps she controls. The opposite is true in non-routine services. We also find that the number of service steps controlled by the provider may change with the extent of interdependencies between the different steps; the direction of the effect rests upon the relative variability that the provider introduces into the service system. Still, our insights come with a cautious statement captured by the term ceteris paribus. The amount of control that the provider should exert by design does not rest solely on the type of the service, but it is moderated by her expertise advantage.

Our conceptualization of the customer experience and the different service elements observed in practice gives form to a useful classification of services. This classification is based on the provider's expertise advantage $\Delta V_{i}$ and the type of the service $\Delta \sigma$ as we have already illustrated in Figure 1. To establish how the optimal design rests upon the joint effect of the provider's expertise advantage and the type of the service, consider 
the following services: i) the lab testing process for routine blood panels (e.g., lipoprotein analyses), ii) the dining service at a theme restaurant, iii) the dining service at a gourmet restaurant, and iv) the lab testing process for a condition of sensitive and private nature (e.g., screening for sexually transmitted diseases).

We can approximately map these services to different points on a replica of Figure 1 (i.e., Figure 10; see points A-D). Lab testing may require a relatively small expertise advantage because of advances in technology (e.g., it may be relatively easy for customers to draw their own sample by using a provided test kit). However, due to fear of embarrassment/social judgment or privacy concerns, customers may consider testing for conditions of sensitive nature (point $\mathrm{C}$ ) to be more experiential than testing of routine blood work (point A). As we can see in Figure 10, the optimal number of steps that the provider controls at point $\mathrm{A}$ is smaller than at point $\mathrm{C}$. This is consistent with practices of providers like myLabBox who delegate more steps of the service to the customers than providers who focus on more routine blood testing.

Figure 10 The interaction of expertise advantage $\left(A_{[1]}\right)$ and service type $(\Delta \sigma)$.

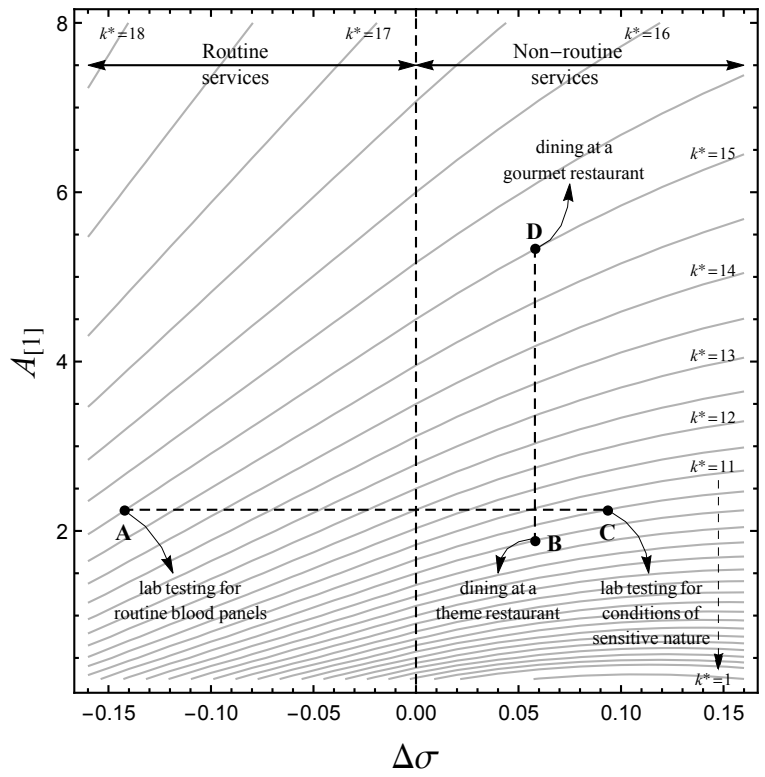

Note. For a given $A_{[n]}$ and $n$, larger values of $A_{[1]}$ imply that the service comprises more steps where the provider has an expertise advantage over the customer (i.e., more steps with $A(k)>0$ and $\Delta V_{i}=V_{i}^{P}-V_{i}^{C}>0$ ). We also remind the reader that $\Delta \sigma \doteq \sigma_{P}-\sigma_{C}=(1-\delta) \sigma_{P}$, where $\delta>1$ for routine services and $\delta \in(0,1)$ for non-routine services. We calculate $k^{*}$ using $n=20, r=0.45, A_{[n]}=-1.5$, and $\rho=0.4$.

Along similar lines, dining at a theme restaurant (point B; e.g., Hard Rock Cafe) and dining at a gourmet restaurant (point D; e.g., a French restaurant) can be considered 
non-routine services (or in relative terms, less routine than dining at more casual establishments) because in both cases, customers may factor in the valuation of the service intangible aspects such as the space ambience, or the employee behavior. Although both services are non-routine, Figure 10 indicates that a gourmet restaurant may allow customers to control a smaller part of the service than a theme restaurant. The reason is that offering such a service entails higher expertise advantage (e.g., knowledge about best combinations of food ingredients, or cooking preparations). This is consistent with known practices of upscale restaurants which restrict customer engagement in their service delivery (Chowhound 2011).

Based on Figure 10, we also see that the optimal service design cannot be based solely on the provider's expertise advantage or the type of the service. Interestingly, the extent of the provider's control may be the same for services as different as dining at a gourmet restaurant and having routine blood tests. Said differently, the provider's control is not simply the result of a larger expertise advantage or a specific service type. It is the interaction of these two dimensions that determines the optimal design. Although conventional wisdom would likely indicate that providers should adopt a full-service approach mainly for services that are non-routine and for which they have high expertise, Figure 10 shows that providers may assume the same (or even more; e.g., consider any point on the left of point A) control of more "prosaic" services.

With respect to the economic aspect of the optimal service design, we find that that services where the steps differ significantly in terms of the provider's expertise or the customers' ability to perform them are always the most profitable. What is more interesting though, is the fact that higher profit does not translate into more control. Depending on the level of expertise more or fewer service steps might be controlled.

Our paper provides some grounding insights into the design of service lines. Although offering a service line always facilitates better market segmentation, it may not always be economically profitable to do so. This is because deploying a service line may introduce costs (e.g., cost of new facilities/equipment or hiring and training of employees) that stem from the fact that services cannot be inventoried. From a profitability point of view, a service line holds more promise for service processes of intermediate length. For smaller or larger services we find that the economic benefit of introducing a service line is less 
pronounced. Finally, we characterize an important insight regarding the role of competition in service design. In particular, we find that competition incentivizes low-capability providers to gravitate towards more control and offer a "super-service" whereas, more capable providers reduce the number of steps they control and provide a "focused service" that targets the high-capability customers.

To obtain first-order insights, our model was developed on the basis of a few simplifying assumptions. Relaxing these assumptions presents opportunities for future research. For instance, future work can focus on more involved cost structures that account for economies of scale, capacity/workforce allocation, congestion effects, or increased rework due to customer involvement. It was also assumed that customers are fully informed about the provider's overall capability. Evaluating whether a provider's control allocation strategy can credibly signal her capability can also provide insights not generated by our model. 


\section{Appendix}

Proof of Lemma 1.The service provider determines the optimal design based on

$$
\begin{aligned}
& \underset{\mathcal{P} \subseteq \mathcal{J}, p \geq 0}{\operatorname{maximize}} \Pi(\mathcal{P}, p)=\left(p-\sum_{i \in \mathcal{P}} c_{i} V_{i}^{P}\right) M \\
& \text { s.t. } \mathbb{E}[U(\mathcal{P}, p)] \geq \mathbb{E}[U(\mathcal{P}=\emptyset, p=0)] .
\end{aligned}
$$

The participation constraint can be rewritten as

$$
\begin{aligned}
& \mathbb{E}[U(\mathcal{P}, p)] \geq \mathbb{E}[U(\mathcal{P}=\emptyset, p=0)] \Leftrightarrow \\
& V(\mathcal{P})-\frac{r}{2} \sigma^{2}(\mathcal{P})-p \geq V(\emptyset)-\frac{r}{2} \sigma^{2}(\emptyset) \Leftrightarrow \\
& p \leq V(\mathcal{P})-V(\emptyset)-\frac{r}{2}\left(\sigma^{2}(\mathcal{P})-\sigma^{2}(\emptyset)\right),
\end{aligned}
$$

where $V(\mathcal{P})=\sum_{i \in \mathcal{P}} V_{i}^{P}+\sum_{i \in \mathcal{C}} V_{i}^{C}$ and $V(\emptyset)=\sum_{i \in \mathcal{J}} V_{i}^{C}$.

Using $k$ to denote the cardinality of $\mathcal{P}$, the variance $\sigma^{2}(\mathcal{P})$ can be calculated as

$$
\begin{aligned}
\sigma^{2}(\mathcal{P}) & =\operatorname{Var}\left[\sum_{i \in \mathcal{P}} \tilde{V}_{i}^{P}+\sum_{i \in \mathcal{C}} \tilde{V}_{i}^{C}\right] \\
& =k \sigma_{P}^{2}+(n-k) \sigma_{C}^{2}+2 \rho\left(\frac{k !}{2 !(k-2) !} \sigma_{P}^{2}+\frac{(n-k) !}{2 !(n-k-2) !} \sigma_{C}^{2}+k(n-k) \sigma_{C} \sigma_{P}\right) \\
& =k(1+(k-1) \rho) \sigma_{P}^{2}+(n-k)(1+(n-k-1) \rho) \sigma_{C}^{2}+2 k(n-k) \rho \sigma_{C} \sigma_{P},
\end{aligned}
$$

where $\sigma_{C} \doteq \delta \sigma_{P}$. Similarly, $\sigma^{2}(\emptyset)=n(1+(n-1) \rho) \sigma_{C}^{2}$ or $\sigma^{2}(\emptyset)=n(1+(n-1) \rho) \delta^{2} \sigma_{P}^{2}$.

We start by deriving the optimal price for a given $\mathcal{P}$ and then we determine the optimal set of steps controlled by the provider. The profit $\Pi(\mathcal{P}, p)$ is linear increasing in $p$. Hence, for a given $\mathcal{P}$, the optimal price is

$$
\begin{aligned}
\tilde{p} & =V(\mathcal{P})-V(\emptyset)-\frac{r}{2}\left(\sigma^{2}(\mathcal{P})-\sigma^{2}(\emptyset)\right) \\
& =\sum_{i \in \mathcal{P}}\left(V_{i}^{P}-V_{i}^{C}\right)-\frac{1}{2}(k r(1-\delta))(1+(k-1) \rho+\delta(1+(2 n-1-k) \rho)) .
\end{aligned}
$$

Define $\tilde{\Pi}(\mathcal{P}) \doteq \Pi(\mathcal{P}, p=\tilde{p})=\sum_{i \in \mathcal{P}} A_{i}-\frac{1}{2}(k r(1-\delta))(1+(k-1) \rho+\delta(1+$ $(2 n-1-k) \rho))$, where $A_{i} \doteq\left(V_{i}^{P}-V_{i}^{C}\right)-c_{i} V_{i}^{P}$. The service provider determines the optimal set of steps to control based on

$$
\underset{\mathcal{P}}{\operatorname{maximize}} \tilde{\Pi}(\mathcal{P})=\sum_{i \in \mathcal{P}} A_{i}-\frac{1}{2}(k r(1-\delta))(1+(k-1) \rho+\delta(1+(2 n-1-k) \rho)) .
$$


We now use an exchange argument to show that the service steps should be assigned in decreasing order of $A_{i}$ for $i \in\{1, \ldots, n\}$. Let $A_{[j]}$ indicate the $j^{\text {th }}$ element of that ordering. Assume that the service provider decides to control $k$ steps. Additionally assume that the provider can use two different configurations (out of the $\frac{n !}{(n-k) !}$ possible configurations) for the choice of the service steps. In the first configuration, the service steps are chosen in a decreasing order of $A_{[i]}$ such that $A_{[j-1]} \geq A_{[j]}$, i.e., $\mathcal{P}_{1}=\left\{A_{[1]}, A_{[2]}, \ldots, A_{[k-1]}, A_{[k]}\right\}$. In the second configuration, the service steps are chosen such that $A_{[j-1]} \geq A_{[j]} \geq A_{[j+w]}$, for any $w \in\{1, \ldots, n-k-1\}$, i.e., $\mathcal{P}_{2}=\left\{A_{[1]}, A_{[2]}, \ldots, A_{[k-1]}, A_{[k+w]}\right\}$. It is straightforward to show that $\Pi\left(\mathcal{P}_{2}\right)-\Pi\left(\mathcal{P}_{1}\right)=A_{[k+w]}-A_{[k]} \leq 0$. Since this holds for any $w$, it proves the claim that the provider's optimal decision is to choose to control the set of steps $\mathcal{P}_{1}$.

Proof of Lemma 2. We assume that the provider's marginal benefit is described by $A(k)=A_{[1]}-\left(A_{[1]}-A_{[n]}\right) k / n$, where $k \in[0, n], A_{[1]}>0$ and $A_{[n]} \leq 0$. We can restate the provider's profit as

$$
\tilde{\Pi}(k)=\int_{0}^{k} A(x) d x-\frac{1}{2}(k r(1-\delta))(1+(k-1) \rho+\delta(1+(2 n-1-k) \rho)) .
$$

It is straightforward to calculate $\int_{0}^{k} A(x) d x=A_{[1]} k-\frac{A_{[1]}-A_{[n]}}{2 n} k^{2}$. The provider determines the optimal number of steps $k^{*}$ she controls based on $\underset{0 \leq k \leq n}{\operatorname{maximize}} \tilde{\Pi}(k)=A_{[1]} k-\frac{A_{[1]}-A_{[n]}}{2 n} k^{2}-\frac{1}{2}(k r(1-\delta))(1+(k-1) \rho+\delta(1+(2 n-1-k) \rho))$. $\tilde{\Pi}(k)$ is concave in $k$ because $\frac{\partial^{2} \tilde{\Pi}(k)}{\partial^{2} k}=-\frac{\left(A_{[1]}-A_{[n]}+n r(1-\delta)^{2} \rho \sigma_{P}^{2}\right)}{n}<0$. Hence, the solution of $\partial \tilde{\Pi}(k) / \partial k=0$ offers the unconstrained maximizer $k_{u n c}=\frac{n\left(2 A_{[1]}-(1-\delta) r \sigma_{P}^{2}(1-\rho+\delta+(2 n-1) \delta \rho)\right)}{2\left(A_{[1]}-A_{[n]}+(1-\delta)^{2} n \rho r \sigma_{P}^{2}\right)}$, and $k^{*}=\max \left\{\min \left\{k_{u n c}, n\right\}, 0\right\}$. After differentiating $k_{u n c}$ we obtain

$$
\begin{aligned}
\partial k_{u n c} / \partial A_{[1]} & =\frac{n\left(r(1-\delta)(1+(1-\rho) \delta+(2 n-1) \rho) \sigma_{P}^{2}-2 A_{[n]}\right)}{2\left(A_{[1]}-A_{[n]}+n r \sigma_{P}^{2}(1-\delta)^{2} \rho\right)^{2}} \\
\partial k_{u n c} / \partial A_{[n]} & =\frac{n\left(2 A_{[1]}-r(1-\delta)(1-\rho+\delta+(2 n-1) \delta \rho) \sigma_{P}^{2}\right)}{2\left(A_{[1]}-A_{[n]}+n r \sigma_{P}^{2}(1-\delta)^{2} \rho\right)^{2}} .
\end{aligned}
$$

- For non-routine services (i.e., for $\Delta \sigma>0 ; \delta \in(0,1)): \partial k_{u n c} / \partial A_{[1]}>0$ and $k_{u n c}-n=$ $\frac{n\left(2 A_{[n]}-r(1-\delta)(1+\delta+(2 n-1-\delta) \rho) \sigma_{P}^{2}\right)}{2\left(A_{[1]}-A_{[n]}+n r \sigma_{P}^{2}(1-\delta)^{2} \rho\right)}<0$ for any $A_{[1]}>0$. Hence, $k_{u n c} \in(0, n)$ iff $A_{[1]}>\bar{A}_{[1]} \doteq$ $\left\{A_{[1]}: k_{u n c}=0\right\}=\left(r(1-\delta)(1-\rho+\delta+(2 n-1) \delta \rho) \sigma_{P}^{2}\right) / 2>0$ otherwise, $k^{*}=0$.

- For routine services (i.e., for $\Delta \sigma<0 ; \delta>1$ ): $\partial k_{u n c} / \partial A_{[n]}>0$ and $k_{u n c}>0$ for any $A_{[1]}>0, A_{[n]} \leq 0$. Hence, $k_{u n c}<n$ iff $A_{[n]}<\bar{A}_{[n]} \doteq\left\{A_{[n]}: k_{u n c}=n\right\}=r(1-\delta)(1+$ $(1-\rho) \delta+(2 n-1) \rho) \sigma_{P}^{2} / 2<0$. 
At $\sigma_{P}^{2}=0$, the difference $k_{u n c}\left(\delta_{1}\right)-k_{u n c}\left(\delta_{2}\right)=0$. Furthermore, $\frac{\partial\left(k_{u n c}\left(\delta_{1}\right)-k_{u n c}\left(\delta_{2}\right)\right)}{\partial \sigma_{P}^{2}}<0$ (analytical expression available upon request) for any $\sigma_{P}^{2}$. Hence, $k_{u n c}\left(\delta_{1}\right)-k_{u n c}\left(\delta_{2}\right)<0$ (i.e., $\left.k_{\text {unc }}\left(\delta_{2}\right)>k_{\text {unc }}\left(\delta_{1}\right)\right)$ always. $\square$

Proof of Corollary 1. Define $\hat{k} \doteq\{k: A(k)=0\}=\frac{n A_{[1]}}{A_{[1]}+A_{[n]}}$. Based on that we derive $\hat{k}-k_{u n c}=\frac{n A_{[1]}}{A_{[1]}+A_{[n]}}-\frac{n\left(2 A_{[1]}(1-\delta) r \sigma_{P}^{2}(1-\rho+\delta+(2 n-1) \delta \rho)\right)}{2\left(A_{[1]}-A_{[n]}+(1-\delta)^{2} n \rho r \sigma_{P}^{2}\right)}$. At $\sigma_{P}^{2}=0$, the difference $\hat{k}-k_{u n c}=$ 0. Furthermore, $\frac{\partial\left(\hat{k}-k_{u n c}\right)}{\partial \sigma_{P}^{2}}=\frac{n r(1-\delta)\left(A_{[1]}(1+(1-\rho) \delta+(2 n-1) \rho)-A_{[n]}(1+\delta-\rho+(2 n-1) \delta \rho)\right)}{2\left(A_{[1]}-A_{[n]}+n r \rho(1-\delta)^{2} \sigma_{P}^{2}\right)^{2}}$ for which it is straightforward to show that $\frac{\partial\left(\hat{k}-k_{u n c}\right)}{\partial \sigma_{P}^{2}}>0$ for all $\delta \in(0,1)$ and $\frac{\partial\left(\hat{k}-k_{u n c}\right)}{\partial \sigma_{P}^{2}}<0$ for all $\delta>1$. Hence, $k_{u n c}<\hat{k}$ for non-routine and $\hat{k}<k_{\text {unc }}$ for routine services.

Proof of Proposition 1. With respect to the comparative statics of the optimal number of steps we focus on the cases where $k^{*}=k_{u n c}$ as in extreme design solutions (i.e., $k^{*}=0$ or $k^{*}=n$ ) any local perturbations leave the design unaffected and bear little insight. Doing so we obtain

$$
\begin{aligned}
\partial k^{*} / \partial n & =\frac{(1-\delta) \rho r \sigma_{P}^{2}\left(A_{[1]}-A_{[n]}\right)(1-\delta(4 n-1))+\left(A_{[1]}-A_{[n]}\right)\left(2 A_{[1]}-\left(1-\delta^{2}\right) r \sigma_{P}^{2}\right)-2 \delta(1-\delta)^{3}\left(n \rho r \sigma_{P}^{2}\right)^{2}}{2\left(A_{[1]}-A_{[n]}+(1-\delta)^{2} n \rho r \sigma_{P}^{2}\right)^{2}} \\
\partial k^{*} / \partial \rho & =\frac{(1-\delta) n r \sigma_{P}^{2}\left(-A_{[1]}(2 n-\delta-1)+A_{[n]}(\delta(2 n-1)-1)+(1+\delta)(1-\delta)^{2} n r \sigma_{P}^{2}\right)}{2\left(A_{[1]}-A_{[n]}+(1-\delta)^{2} n \rho r \sigma_{P}^{2}\right)^{2}}
\end{aligned}
$$

- For non-routine services (i.e., for $\Delta \sigma>0 ; \delta \in(0,1)): \partial k^{*} / \partial n>0$ iff $n<\hat{n} \doteq$ $-\frac{A_{[1]}-A_{[n]}}{r \sigma_{P}^{2} \rho(1-\delta)^{2}}+\sqrt{\frac{\left(A_{[1]}-A_{[n]}\right)\left(2\left(A_{[1]}-\delta A_{[n]}\right)-r \sigma_{P}^{2}(1-\delta)^{2}(1+\delta)(1-\rho)\right)}{2 \sigma_{P}^{2}\left(r(1-\delta)^{2} \delta \rho\right)^{2}}}$, and $\partial k^{*} / \partial \rho<0$ iff $\sigma_{P}^{2}<\hat{\sigma}_{P_{1}}^{2} \doteq$ $\frac{A_{[1]}(2 n-1-\delta)+A_{[n]}(1-\delta(2 n-1))}{n r(1-\delta)^{2}(1+\delta)}$.

- For routine services (i.e., when $\Delta \sigma<0 ; \delta>1$ ): $\partial k^{*} / \partial n>0$, and $\partial k^{*} / \partial \rho>0$ iff $\sigma_{P}^{2}<$ $\hat{\sigma}_{P_{2}}^{2} \doteq \frac{A_{[1]}(2 n-1-\delta)+A_{[n]}(1-\delta(2 n-1))}{n r(1-\delta)^{2}(1+\delta)}$.

Proof of Proposition 2. We adopt the notation $k^{*}(|\mathcal{J}|)$ and $\Pi^{*}(|\mathcal{J}|)$ to indicate the optimal number of steps and the optimal profit, when the service process is homogeneous with $A(k)=\frac{A_{[1]}+A_{[n]}}{2}$. For a heterogeneous process we use the notation $k^{*}(\mathcal{J})$ and $\Pi^{*}(\mathcal{J})$. Similar to the proof of Lemma 2 we derive $k_{u n c}(|\mathcal{J}|)=\frac{A_{[1]}+A_{[n]}-r(1-\delta) \sigma_{P}^{2}(1+\delta+(\delta(2 n-1)-1) \rho)}{2 r \rho(1-\delta)^{2} \sigma_{P}^{2}}$. Then,

- For non-routine services (i.e., for $\Delta \sigma>0 ; \delta \in(0,1))$ : $k_{u n c}(|\mathcal{J}|) \in(0, n)$ iff $A_{[1]} \in$ $\left(2 \bar{A}_{[1]}-A_{[n]}, 2 \bar{A}_{[1]}-A_{[n]}+2 r\left(1-\delta^{2}\right)(1+(n-1) \rho) \sigma_{P}^{2}\right)$.

- For routine services (i.e., for $\Delta \sigma<0 ; \delta>1)$ : $k_{\text {unc }}(|\mathcal{J}|) \in(0, n)$ iff $A_{[n]} \in$ $\left(2 \bar{A}_{[n]}-A_{[1]}-2 r\left(1-\delta^{2}\right)(1+(n-1) \rho) \sigma_{P}^{2}, 2 \bar{A}_{[n]}-A_{[1]}\right)$. 
We focus on $k^{*}(|\mathcal{J}|) \in(0, n)$, which also implies $k^{*}(\mathcal{J}) \in(0, n)$ because $2 \bar{A}_{[1]}-A_{[n]}+$ $2 r\left(1-\delta^{2}\right)(1+(n-1) \rho) \sigma_{P}^{2}>\bar{A}_{[1]}$ and $2 \bar{A}_{[n]}-A_{[1]}<A_{[n]}$. Based on $k^{*}(|\mathcal{J}|)$ and $p^{*}(|\mathcal{J}|)=$ $\tilde{p}\left(k=k^{*}(|\mathcal{J}|)\right)$, we calculate the provider's profit in a homogeneous process as

$$
\Pi^{*}(|\mathcal{J}|)=\frac{\left(A_{[1]}+A_{[n]}-r(1-\delta) \sigma_{P}^{2}(1+\delta+(\delta(2 n-1)-1) \rho)\right)^{2}}{8 r \rho(1-\delta)^{2} \sigma_{P}^{2}} .
$$

The profit differential

$$
\begin{gathered}
\Pi^{*}(|\mathcal{J}|)-\Pi^{*}(\mathcal{J})=\frac{\left(A_{[1]}+A_{[n]}-r(1-\delta) \sigma_{P}^{2}(1+\delta+(\delta(2 n-1)-1) \rho)\right)^{2}}{8 r \rho(1-\delta)^{2} \sigma_{P}^{2}} \\
-\frac{n\left(2 A_{[1]}-r(1-\delta) \sigma_{P}^{2}(1+\delta+(\delta(2 n-1)-1) \rho)\right)^{2}}{8\left(A_{[1]}-A_{[n]}+(1-\delta)^{2} n \rho r \sigma_{P}^{2}\right)}<0 \text { for } \\
A_{[1]} \in\left(2 \bar{A}_{[1]}-A_{[n]}, 2 \bar{A}_{[1]}-A_{[n]}+2 r\left(1-\delta^{2}\right)(1+(n-1) \rho) \sigma_{P}^{2}\right) \text { when } \Delta \sigma>0, \\
A_{[n]} \in\left(2 \bar{A}_{[n]}-A_{[1]}-2 r\left(1-\delta^{2}\right)(1+(n-1) \rho) \sigma_{P}^{2}, 2 \bar{A}_{[n]}-A_{[1]}\right) \text { when } \Delta \sigma<0 .
\end{gathered}
$$

Furthermore, $k^{*}(|\mathcal{J}|)-k^{*}(\mathcal{J})=\frac{\left(A_{[1]}-A_{[n]}\right)\left(A_{[1]}+A_{[n]}-r\left(1-\delta^{2}\right) \sigma_{P}^{2}(1+(n-1) \rho)\right)}{2(1-\delta)^{2} \rho r \sigma_{P}^{2}\left(A_{[1]}-A_{[n]}+(1-\delta)^{2} n \rho r \sigma_{P}^{2}\right)}>0$ iff

$$
\begin{aligned}
& \left.A_{[1]}>\hat{A}_{[1]} \doteq A_{[1]}+A_{[n]}-r\left(1-\delta^{2}\right)(1+(n-1) \rho) \sigma_{P}^{2} \text { when } \Delta \sigma>0 \text { (i.e., when } \delta \in(0,1)\right), \\
& \left.A_{[1]}>\tilde{A}_{[1]} \doteq A_{[1]}+A_{[n]}-r\left(1-\delta^{2}\right)(1+(n-1) \rho) \sigma_{P}^{2} \text { when } \Delta \sigma<0 \text { (i.e., when } \delta>1\right) .
\end{aligned}
$$

It is straightforward to show that $\tilde{A}_{[1]}<\hat{A}_{[1]}$.

Proof of Proposition 3. In the remaining of the proofs we make use of the average quantities $\bar{V}^{P}, \bar{V}^{C}, \bar{c}$ and consider only the meaningful case of positive marginal benefit $\left(\bar{V}^{P}-\bar{V}^{C}\right)-\bar{c} \bar{V}^{P}>0$ (i.e., the average marginal benefit that the provider can derive is positive). We capture a heterogeneous customer population by normalizing the total size of the market to one and by assuming the different types of customers $\theta$ to be distributed according to $\bar{V}^{C}(\theta) \sim \mathcal{U}\left[0, \bar{V}^{P}\right]$. The marginal customer $(\hat{\theta})$ holds a capability $\bar{V}^{C}(\hat{\theta}) \doteq$ $\left\{\bar{V}^{C}(\theta): \mathbb{E}[U(\mathcal{P}, p)]-\mathbb{E}[U(\mathcal{P}=\emptyset, p=0)]=0\right\}$ is indifferent between receiving the service from the provider and entirely self-performing it. The consumer surplus $\mathbb{E}[U(\mathcal{P}, p)]-$ $\mathbb{E}[U(\mathcal{P}=\emptyset, p=0)]$ decreases in $\bar{V}^{C}$ as $\frac{\partial(\mathbb{E}[U(\mathcal{P}, p)]-\mathbb{E}[U(\mathcal{P}=\emptyset, p=0)])}{\partial V^{C}}=-k<0$. Therefore, the provider serves the market

$$
M(p, k) \doteq \frac{\bar{V}^{C}(\hat{\theta})}{\bar{V}^{P}}=1-\frac{p+\frac{1}{2} k r(1-\delta)(1+(k-1) \rho+\delta(1-\rho+(2 n-k) \rho)) \sigma_{P}^{2}}{k \bar{V}^{P}}
$$


The provider's maximization problem is given by

$$
\begin{gathered}
\underset{0 \leq k \leq n, p \geq 0}{\operatorname{maximize}} \Pi=\left(p-k \bar{c} \bar{V}^{P}\right) M(p, k) \\
\text { s.t } \quad 0 \leq M(p, k) \leq 1 .
\end{gathered}
$$

By differentiating $\Pi$, we obtain $\partial^{2} \Pi / \partial^{2} p=-\frac{2}{k V^{P}}<0$. Hence, solving $\partial \Pi / \partial p=0$ for $p$ returns the optimal price (for a given $k$ )

$$
\tilde{p}=\frac{1}{4} k\left(2(1+\bar{c}) \bar{V}^{P}-r(1-\delta)(1-(k-1) \rho-\delta(1-\rho-(2 n-k) \rho))\right) \sigma_{P}^{2} .
$$

We define

$$
\tilde{\Pi}(k) \doteq \Pi(k, p=\tilde{p})=\frac{k\left(2(1-\bar{c}) \bar{V}^{P}+r(1-\delta) \sigma_{P}^{2}(1+(k-1) \rho+\delta(1+(2 n-1-k) \rho))\right)^{2}}{16 \bar{V}^{P}} .
$$

The provider maximizes $\tilde{\Pi}$ subject to $0 \leq k \leq n$ and $\tilde{M}(k) \doteq M(p=\tilde{p}, k) \in[0,1]$, where

$$
M(p=\tilde{p}, k)=\frac{2(1-\bar{c}) \bar{V}^{P}+r(1-\delta) \sigma_{P}^{2}(1+(k-1) \rho+\delta(1+(2 n-1-k) \rho))}{4 \bar{V}^{P}} .
$$

The Lagrangean of the provider's problem is given by

$$
\mathcal{L}=\tilde{\Pi}+\lambda_{1}(n-k)+\lambda_{2} k+\lambda_{3}(1-\tilde{M})+\lambda_{4} \tilde{M} .
$$

To determine the optimal design we identify the critical points that satisfy the Kuhn-Tucker conditions: $\partial \mathcal{L} / \partial k=0, \partial \mathcal{L} / \partial \lambda_{1} \geq 0, \partial \mathcal{L} / \partial \lambda_{2} \geq 0, \partial \mathcal{L} / \partial \lambda_{3} \geq 0, \partial \mathcal{L} / \partial \lambda_{4} \geq 0, \lambda_{1} \partial \mathcal{L} / \partial \lambda_{1}=0$, $\lambda_{2} \partial \mathcal{L} / \partial \lambda_{2}=0, \lambda_{3} \partial \mathcal{L} / \partial \lambda_{3}=0, \lambda_{4} \partial \mathcal{L} / \partial \lambda_{4}=0, \lambda_{1} \geq 0, \lambda_{2} \geq 0, \lambda_{3} \geq 0$, and $\lambda_{4} \geq 0$.

- For non-routine services (i.e., for $\Delta \sigma>0 ; \delta \in(0,1))$ we obtain:

i) When $\sigma_{P}^{2} \in\left(0, \frac{2(1-\bar{c}) \bar{V}^{P}}{r(1-\delta)(1+(3 n-1) \rho+\delta-(n+1) \delta \rho)}\right]$

$$
k^{*}=n \text { and } \Pi^{*}=\frac{n\left(2(1-\bar{c}) \bar{V}^{P}-r\left(1-\delta^{2}\right)(1+(n-1) \rho) \sigma_{P}^{2}\right)^{2}}{16 \bar{V}^{P}} .
$$

ii) When $\sigma_{P}^{2} \geq \frac{2(1-\bar{c}) \bar{V}^{P}}{r(1-\delta)(1-\rho+\delta+(2 n-1) \delta \rho)}, k^{*}=0$ and $\Pi^{*}=0$.

iii) When $\sigma_{P}^{2} \in\left(\frac{2(1-\bar{c}) \bar{V}^{P}}{r(1-\delta)(1+(3 n-1) \rho+\delta-(n+1) \delta \rho)}, \frac{2(1-\bar{c}) \bar{V}^{P}}{r(1-\delta)(1-\rho+\delta+(2 n-1) \delta \rho)}\right)$

$$
\begin{aligned}
k^{*} & =\frac{2(1-\bar{c}) \bar{V}^{P}-r(1-\delta)(1+\delta-\rho+(2 n-1) \delta \rho) \sigma_{P}^{2}}{3 r(1-\delta)^{2} \rho \sigma_{P}^{2}} \in(0, n) \\
\Pi^{*} & =\frac{\left(2(1-\bar{c}) \bar{V}^{P}-r(1-\delta)(1+\delta-\rho+(2 n-1) \delta \rho)\right)^{3}}{108 r \bar{V}^{P}(1-\delta)^{2} \rho \sigma_{P}^{2}} .
\end{aligned}
$$

In this case, $\partial^{2} \tilde{\Pi} /\left.\partial^{2} k\right|_{k=k^{*}}=-\frac{r(1-\delta)^{2} \rho \sigma_{P}^{2}\left(2(1-\bar{c}) \bar{V}^{P}-r(1-\delta)(1-\rho+\delta+(2 n-1) \delta \rho) \sigma_{P}^{2}\right)}{8 \bar{V}^{P}}<0$. 
- For routine services (i.e., for $\Delta \sigma<0 ; \delta>1$ ) we obtain: $k^{*}=n$ and $\Pi^{*}=$ $\frac{n\left(2(1-\bar{c}) \bar{V}^{P}-r\left(1-\delta^{2}\right)(1+(n-1) \rho) \sigma_{P}^{2}\right)^{2}}{16 \bar{V}^{P}}$ for any $\sigma_{P}^{2}$.

In the case of a homogeneous market, we assume that all customers have the same type $|\theta|$. In particular, as we mention in the main paper, the customers of the homogeneous market have the same capability $\bar{V}^{C}=\bar{V}^{P} / 2$ as the "average" customer of the heterogeneous market (we also assume $\bar{c}<1 / 2$ so that the marginal benefit $(1 / 2-\bar{c}) \bar{V}^{P}>0$ ). The provider's problem can written as

$$
\underset{0 \leq k \leq n}{\operatorname{maximize}} \tilde{\Pi}(k)=\frac{k}{2}\left((1-2 \bar{c}) \bar{V}^{P}-r(1-\delta) \sigma_{P}^{2}(1+(k-1) \rho+\delta(1+(2 n-1-k) \rho))\right) .
$$

Following the same steps as in the proof of Lemma 2 we obtain

$$
\begin{aligned}
& k_{u n c}=\frac{\left((1-2 \bar{c}) \bar{V}^{P}-(1-\delta) r \sigma_{P}^{2}(1-\rho+\delta+(2 n-1) \delta \rho)\right)^{2}}{8(1-\delta)^{2} n \rho \sigma_{P}^{2}} \\
& \Pi_{u n c}\left(k=k_{u n c} ;|\theta|\right)=\frac{\left((1-2 \bar{c}) \bar{V}^{P}-r(1-\delta) \sigma_{P}^{2}(1+\delta+(\delta(2 n-1)-1) \rho)\right)^{2}}{8 r \rho(1-\delta)^{2} \sigma_{P}^{2}} .
\end{aligned}
$$

- For non-routine services (i.e., for $\Delta \sigma>0 ; \delta \in(0,1))$ :

i) When $\sigma_{p}^{2} \geq \frac{(1-2 \bar{c}) \bar{V}^{P}}{r(1-\delta)(1-\rho+\delta+(2 n-1) \delta \rho)}, k^{*}=0$, and $\Pi^{*}=0$.

ii) When $\sigma_{p}^{2} \in\left(0, \frac{(1-2 \bar{c}) \bar{V}^{P}}{r(1-\delta)(1+(1-\rho) \delta+(2 n-1) \rho)}\right]$,

$$
k^{*}=n \text { and } \Pi^{*}=\frac{n}{2}\left((1-2 \bar{c}) \bar{V}^{P}-r\left(1-\delta^{2}\right)(1+(n-1) \rho) \sigma_{P}^{2}\right) .
$$

iii) When $\sigma_{P}^{2} \in\left(\frac{(1-2 \bar{c}) \bar{V}^{P}}{r(1-\delta)(1+(1-\rho) \delta+(2 n-1) \rho)}, \frac{\left(1-2 \bar{c} \bar{V}^{P}\right.}{r(1-\delta)(1-\rho+\delta+(2 n-1) \delta \rho)}\right), k^{*}=k_{u n c}, \Pi^{*}=\Pi_{u n c}$.

- For routine services (i.e., for $\Delta \sigma<0 ; \delta>1$ ):

$$
k^{*}=n \text { and } \Pi^{*}=\frac{n}{2}\left((1-2 \bar{c}) \bar{V}^{P}-r\left(1-\delta^{2}\right)(1+(n-1) \rho) \sigma_{P}^{2}\right) \text { for any } \sigma_{P}^{2} .
$$

To establish the existence of a switching point before/after which the provider benefits more from offering the service under a heterogeneous or homogenous market we compare the corresponding profits.

- For non-routine services (i.e., for $\Delta \sigma>0 ; \delta \in(0,1)$ ) we focus in the region $\sigma_{P}^{2} \in$ $\left(\frac{2(1-\bar{c}) \bar{V}^{P}}{r(1-\delta)(1+(3 n-1) \rho+\delta-(n+1) \delta \rho)}, \frac{(1-2 \bar{c}) \bar{V}^{P}}{r(1-\delta)(1-\rho+\delta+(2 n-1) \delta \rho)}\right)$ where

- Under a heterogeneous market:

$$
\begin{aligned}
k^{*} & =\frac{2(1-\bar{c}) \bar{V}^{P}-r(1-\delta)(1+\delta-\rho+(2 n-1) \delta \rho) \sigma_{P}^{2}}{3 r(1-\delta)^{2} \rho \sigma_{P}^{2}} \in(0, n) \\
\Pi^{*} & =\frac{\left(2(1-\bar{c}) \bar{V}^{P}-r(1-\delta)(1+\delta-\rho+(2 n-1) \delta \rho)\right)^{3}}{108 r \bar{V}^{P}(1-\delta)^{2} \rho \sigma_{P}^{2}} .
\end{aligned}
$$


- Under a homogeneous market:

$$
\begin{aligned}
& k^{*}(|\theta|)=\frac{\left((1-2 \bar{c}) \bar{V}^{P}-(1-\delta) r \sigma_{P}^{2}(1-\rho+\delta+(2 n-1) \delta \rho)\right)^{2}}{8(1-\delta)^{2} n \rho \sigma_{P}^{2}} \in(0, n) \\
& \Pi^{*}(|\theta|)=\frac{\left((1-2 \bar{c}) \bar{V}^{P}-r(1-\delta) \sigma_{P}^{2}(1+\delta+(\delta(2 n-1)-1) \rho)\right)^{2}}{8 r \rho(1-\delta)^{2} \sigma_{P}^{2}} .
\end{aligned}
$$

Based on the above, we find that $\Pi^{*}-\Pi^{*}(|\theta|)>0$ iff $\sigma_{P}^{2}>\frac{2(1-4 \bar{c}) \bar{V}^{P}}{r(1-\delta)(1+\delta-\rho+(2 n-1) \delta \rho)} \doteq \bar{\sigma}_{P}^{2}$, where $\bar{\sigma}_{P}^{2} \in\left(\frac{2(1-\bar{c}) \bar{V}^{P}}{r(1-\delta)(1+(3 n-1) \rho+\delta-(n+1) \delta \rho)}, \frac{(1-2 \bar{c}) \bar{V}^{P}}{r(1-\delta)(1-\rho+\delta+(2 n-1) \delta \rho)}\right)$.

- For routine services (i.e., for $\Delta \sigma<0 ; \delta>1$ ) we compare

$$
\begin{aligned}
& \Pi^{*}=\frac{n\left(2(1-\bar{c}) \bar{V}^{P}-r\left(1-\delta^{2}\right)(1+(n-1) \rho) \sigma_{P}^{2}\right)^{2}}{16 \bar{V}^{P}} \text { with } \\
& \Pi^{*}(|\theta|)=\frac{n}{2}\left((1-2 \bar{c}) \bar{V}^{P}-r\left(1-\delta^{2}\right)(1+(n-1) \rho) \sigma_{P}^{2}\right),
\end{aligned}
$$

and we find that $\Pi^{*}-\Pi^{*}(|\theta|)>0$ iff $\sigma_{P}^{2}<\frac{2(1-\sqrt{2}+\bar{c}) \bar{V}^{P}}{r\left(\delta^{2}-1\right)(1+(n-1) \rho)} \doteq \overline{\bar{\sigma}}_{P}^{2}$

Proof of Proposition 4. We now consider the case where the provider designs a service line comprising two services. In particular, the provider designs a service that targets the lower end of the market by controlling $k_{0}$ steps and charging $p_{0}$, and a different service that targets the higher end of the market by controlling $k_{1} \neq k_{0}$ steps and charging $p_{1} \neq p_{0}$. The $\hat{\theta}_{0}$ customer with a capability $\bar{V}^{C}\left(\hat{\theta}_{0}\right) \doteq\left\{\bar{V}^{C}(\theta): \mathbb{E}\left[U\left(\mathcal{P}_{0}, p_{0}\right)\right]-\mathbb{E}\left[U\left(\mathcal{P}_{1}, p_{1}\right)\right]=0\right\}$ is indifferent between the two services. Similarly, the $\hat{\theta}_{1}$ customer with a capability $\bar{V}^{C}\left(\hat{\theta}_{1}\right) \doteq\left\{\bar{V}^{C}(\theta): \mathbb{E}\left[U\left(\mathcal{P}_{1}, p_{1}\right)\right]-\mathbb{E}[U(\mathcal{P}=\emptyset, p=0)]=0\right\}$ is indifferent between receiving the service from the provider and entirely self-performing it. The number of customers that the provider serves through the $\left(k_{0}, p_{0}\right)$ service is

$$
\begin{aligned}
M_{0}\left(p_{0}, k_{0}, p_{1}, k_{1}\right) \doteq \frac{\bar{V}^{C}\left(\hat{\theta}_{0}\right)}{\bar{V}^{P}} & =1-\frac{p_{1}-p_{0}}{\left(k_{1}-k_{0}\right) \bar{V}^{P}} \\
& +\frac{r(1-\delta)\left(1+\left(k_{0}+k_{1}-1\right) \rho+\delta\left(1+\left(2 n-k_{0}-k_{1}-1\right) \rho\right)\right) \sigma_{P}^{2}}{2 \bar{V}^{P}} .
\end{aligned}
$$

The number of customers served through the $\left(k_{1}, p_{1}\right)$ service is

$$
M_{1}\left(p_{0}, k_{0}, p_{1}, k_{1}\right) \doteq \frac{\bar{V}^{C}\left(\hat{\theta}_{1}\right)-\bar{V}^{C}\left(\hat{\theta}_{0}\right)}{\bar{V}^{P}}=\frac{\frac{2\left(k_{0} p-k p_{0}\right)}{k_{1}^{2}-k_{1} k_{0}}+r k_{0}(1-\delta)^{2} \rho \sigma_{P}^{2}}{2 \bar{V}^{P}} .
$$

The provider's maximization problem is given by

$$
\begin{gathered}
\underset{0 \leq k_{0}, k_{1} \leq n, p_{0}, p_{1} \geq 0}{\operatorname{maximize}} \Pi_{\text {line }}=\left(p_{0}-k_{0} \bar{c} \bar{V}^{P}\right) M_{0}+\left(p_{1}-k_{1} \bar{c} \bar{V}^{P}\right) M_{1} \\
\text { s.t } \quad M_{0} \geq 0, M_{1} \geq 0 \\
\\
M_{0}+M_{1} \leq 1 .
\end{gathered}
$$


By differentiating $\Pi_{\text {line }}$, we obtain $\partial^{2} \Pi_{\text {line }} / \partial^{2} p_{0}=\frac{2}{\left(k_{1}-k_{0}\right) \bar{V}^{P}}<0$ iff $k_{1}<k_{0}, \partial^{2} \Pi_{\text {line }} / \partial^{2} p_{1}=$ $\frac{2 k_{0}}{\left(k_{1}-k_{0}\right) k_{1} \bar{V}^{P}}<0$ iff $k_{1}<k_{0}$, and $\partial^{2} \Pi_{\text {line }} / \partial^{2} p_{0} \partial^{2} \Pi_{\text {line }} / \partial^{2} p_{1}-\left(\partial^{2} \Pi_{\text {line }} / \partial p_{1} \partial p_{0}\right)^{2}=\frac{4}{k\left(k_{0}-k_{1}\right) \bar{V}^{P}}>$ 0 iff $k_{1}<k_{0}$ (we show later that $k_{1}^{*}<k_{0}^{*}$ ). Hence, solving $\partial \Pi_{\text {line }} / \partial p_{0}=0$ and $\partial \Pi_{\text {line }} / \partial p_{1}=0$ with respect to $p_{0}$ and $p_{1}$ returns the optimal prices (for given $k_{0}, k_{1}$ )

$$
\begin{aligned}
& \tilde{p}_{0}=\frac{1}{4} k_{0}\left(2(1+\bar{c}) \bar{V}^{P}-r(1-\delta)\left(1-\left(k_{0}-1\right) \rho-\delta\left(1-\rho-\left(2 n-k_{0}\right) \rho\right)\right)\right) \sigma_{P}^{2} \\
& \tilde{p}_{1}=\frac{1}{4} k_{1}\left(2(1+\bar{c}) \bar{V}^{P}-r(1-\delta)\left(1-\left(k_{1}-1\right) \rho-\delta\left(1-\rho-\left(2 n-k_{1}\right) \rho\right)\right)\right) \sigma_{P}^{2} .
\end{aligned}
$$

Based on $\tilde{p}_{0}, \tilde{p}_{1}$ we define $\tilde{\Pi}_{\text {line }}\left(k_{0}, k_{1}\right) \doteq \Pi_{\text {line }}\left(k_{0}, k_{1}, p_{0}=\tilde{p}_{0}, p_{1}=\tilde{p}_{1}\right)$ (analytical expression available upon request). The provider maximizes $\tilde{\Pi}_{\text {line }}$ subject to $0 \leq k_{0}, k_{1} \leq n$, $\tilde{M}_{0}\left(k_{0}, k_{1}\right) \doteq M_{0}\left(k_{0}, k_{1}, p_{0}=\tilde{p}_{0}, p_{1}=\tilde{p}_{1}\right) \geq 0, \tilde{M}_{1}\left(k_{0}, k_{1}\right) \doteq M_{1}\left(k_{0}, k_{1}, p_{0}=\tilde{p}_{0}, p_{1}=\tilde{p}_{1}\right) \geq 0$, and $\tilde{M}_{0}\left(k_{0}, k_{1}\right)+\tilde{M}_{1}\left(k_{0}, k_{1}\right) \leq 1$. The Lagrangean of the provider's problem is given by

$$
\mathcal{L}_{\text {line }}=\tilde{\Pi}_{\text {line }}+\lambda_{1}\left(n-k_{0}\right)+\lambda_{2} k_{0}+\lambda_{3} \tilde{M}_{0}+\mu_{1}\left(n-k_{1}\right)+\mu_{2} k_{1}+\mu_{3} \tilde{M}_{1}+\psi\left(1-\tilde{M}_{0}-\tilde{M}_{1}\right)
$$

We determine the optimal design by identifying the critical points that satisfy the Kuhn-Tucker conditions: $\partial \mathcal{L}_{\text {line }} / \partial k_{0}=0, \partial \mathcal{L}_{\text {line }} / \partial k_{1}=0, \partial \mathcal{L}_{\text {line }} / \partial \lambda_{1} \geq 0, \partial \mathcal{L}_{\text {line }} / \partial \lambda_{2} \geq$ $0, \partial \mathcal{L}_{\text {line }} / \partial \lambda_{3} \geq 0, \partial \mathcal{L}_{\text {line }} / \partial \mu_{1} \geq 0, \partial \mathcal{L}_{\text {line }} / \partial \mu_{2} \geq 0, \partial \mathcal{L}_{\text {line }} / \partial \mu_{3} \geq 0, \partial \mathcal{L}_{\text {line }} / \partial \psi \geq 0$, $\lambda_{1} \partial \mathcal{L}_{\text {line }} / \partial \lambda_{1}=0, \lambda_{2} \partial \mathcal{L}_{\text {line }} / \partial \lambda_{2}=0, \lambda_{3} \partial \mathcal{L}_{\text {line }} / \partial \lambda_{3}=0, \mu_{1} \partial \mathcal{L}_{\text {line }} / \partial \mu_{1}=0, \mu_{2} \partial \mathcal{L}_{\text {line }} / \partial \mu_{2}=0$, $\mu_{3} \partial \mathcal{L}_{\text {line }} / \partial \mu_{3}=0, \psi \partial \mathcal{L}_{\text {line }} / \partial \psi=0, \lambda_{1} \geq 0, \lambda_{2} \geq 0, \lambda_{3} \geq 0, \mu_{1} \geq 0, \mu_{2} \geq 0, \mu_{3} \geq 0$, and $\psi \geq 0$.

- For non-routine services (i.e., for $\Delta \sigma>0 ; \delta \in(0,1)$ ):

i) When $\sigma_{P}^{2} \in\left(0, \frac{2(1-\bar{c}) \bar{V}^{P}}{r(1-\delta)(2(1-\rho+\delta)-(n+2) \delta \rho+5 n \rho)}\right]$, and $k_{1}^{*}=n / 2, k_{0}^{*}=n$.

ii) When $\sigma_{P}^{2} \in\left(\frac{2(1-\bar{c}) \bar{V}^{P}}{r(1-\delta)(2(1-\rho+\delta)-(n+2) \delta \rho+5 n \rho)}, \frac{2(1-\bar{c}) \bar{V}^{P}}{r(1-\delta)(1-\rho+\delta+(2 n-1) \delta \rho)}\right)$,

$$
k_{1}^{*}=\frac{2(1-\bar{c}) \bar{V}^{P}-r(1-\delta)(1-\rho+\delta+(2 n-1) \delta \rho) \sigma_{P}^{2}}{5 r(1-\delta)^{2} \rho \sigma_{P}^{2}}, \text { and } k_{0}^{*}=2 k_{1}^{*} .
$$

iii) When $\sigma_{P}^{2} \geq \frac{2(1-\bar{c}) \bar{V}^{P}}{r(1-\delta)(1-\rho+\delta+(2 n-1) \delta \rho)}, k_{1}^{*}=k_{0}^{*}=0$.

- For routine services (i.e., for $\Delta \sigma<0 ; \delta>1$ ):

i) When $\sigma_{P}^{2} \in\left(0, \frac{2(1+\bar{c}) \bar{V}^{P}}{r(\delta-1)(2(1-\rho+\delta)+n \rho+(3 n-2) \delta \rho)}\right), k_{1}^{*}=n / 2$, and $k_{0}^{*}=n$.

ii) When $\sigma_{P}^{2} \geq \frac{2(1+\bar{c}) \bar{V}^{P}}{r(\delta-1)(2(1-\rho+\delta)+n \rho+(3 n-2) \delta \rho)}$

$$
k_{1}^{*}=\frac{-2(1+\bar{c}) \bar{V}^{P}+r(\delta-1)(1-\rho+\delta+(2 n-1) \delta \rho) \sigma_{P}^{2}}{r(1-\delta)^{2} \rho \sigma_{P}^{2}}, k_{0}^{*}=n \text { and } \psi>0 .
$$


For all cases, we verify sufficiency $\partial^{2} \tilde{\Pi}_{\text {line }} /\left.\partial^{2} k_{0}\right|_{k_{0}=k_{0}^{*}, k_{1}=k_{1}^{*}}, \partial^{2} \tilde{\Pi}_{l i n e} /\left.\partial^{2} k_{1}\right|_{k_{0}=k_{0}^{*}, k_{1}=k_{1}^{*}}<$ 0 , and $\left.\left(\partial^{2} \tilde{\Pi}_{\text {line }} / \partial^{2} k_{0} \partial^{2} \tilde{\Pi}_{\text {line }} / \partial^{2} k_{1}-\left(\partial^{2} \tilde{\Pi}_{\text {line }} / \partial k_{1} \partial k_{0}\right)^{2}\right)\right|_{k_{0}=k_{0}^{*}, k_{1}=k_{1}^{*}}>0$ (analytical expressions available upon request).

To determine the benefit of providing a line of two services over a single service we compare the profitability of the optimal designs that the provider employs under the different $\sigma_{P}^{2}$ ranges we derived above and in the proof of Proposition 3.

- For non-routine services (i.e., for $\Delta \sigma>0 ; \delta \in(0,1)$ ):

i) In the range of $\sigma_{P}^{2} \in\left(0, \frac{2(1-\bar{c}) \bar{V}^{P}}{r(1-\delta)(1+(3 n-1) \rho+\delta-(n+1) \delta \rho)}\right]$ we compare the profits $\tilde{\Pi}_{\text {line }}\left(k_{0}^{*}=n, k_{1}^{*}=n / 2\right)$ and $\tilde{\Pi}\left(k^{*}=n\right)$.

ii) In the range of $\sigma_{P}^{2} \in\left(\frac{2(1-\bar{c}) \bar{V}^{P}}{r(1-\delta)(1+(3 n-1) \rho+\delta-(n+1) \delta \rho)}, \frac{2(1-\bar{c}) \bar{V}^{P}}{r(1-\delta)(2(1-\rho+\delta)-(n+2) \delta \rho+5 n \rho)}\right)$ we compare $\tilde{\Pi}_{\text {line }}\left(k_{0}^{*}=n, k_{1}^{*}=n / 2\right)$ and $\tilde{\Pi}\left(k^{*}=\frac{2(1-\bar{c}) \bar{V}^{P}-r(1-\delta)(1+\delta-\rho+(2 n-1) \delta \rho) \sigma_{P}^{2}}{3 r(1-\delta)^{2} \rho \sigma_{P}^{2}}\right)$.

iii) In the range of $\sigma_{P}^{2} \in\left[\frac{2(1-\bar{c}) \bar{V}^{P}}{r(1-\delta)(2(1-\rho+\delta)-(n+2) \delta \rho+5 n \rho)}, \frac{2(1-\bar{c}) \bar{V}^{P}}{r(1-\delta)(1-\rho+\delta+(2 n-1) \delta \rho)}\right)$ we compare the profits $\tilde{\Pi}_{\text {line }}\left(k_{0}^{*}=2 k_{1}^{*}, k_{1}^{*}=\frac{2(1-\bar{c}) \bar{V}^{P}-r(1-\delta)(1-\rho+\delta+(2 n-1) \delta \rho) \sigma_{P}^{2}}{5 r(1-\delta)^{2} \rho \sigma_{P}^{2}}\right)$ and $\tilde{\Pi}\left(k^{*}=\frac{2(1-\bar{c}) \bar{V}^{P}-r(1-\delta)(1+\delta-\rho+(2 n-1) \delta \rho) \sigma_{P}^{2}}{3 r(1-\delta)^{2} \rho \sigma_{P}^{2}}\right)$.

It is easy to show that $\tilde{\Pi}\left(k_{0}^{*}, k_{1}^{*}\right)-\tilde{\Pi}\left(k^{*}\right)>0$ for all $\sigma_{P}^{2} \in\left(0, \frac{2(1-\bar{c}) \bar{V}^{P}}{r(1-\delta)(1-\rho+\delta+(2 n-1) \delta \rho)}\right)$, which implies that the provider always finds it optimal to offer a line of two services over a single service. To determine how the magnitude of this benefit changes with respect to the length $n$ of the service process, we calculate $\partial\left(\tilde{\Pi}_{\text {line }}\left(k_{0}^{*}, k_{1}^{*}\right)-\tilde{\Pi}\left(k^{*}\right)\right) / \partial n$ (analytical expression available upon request) and we find that $\partial\left(\tilde{\Pi}_{\text {line }}\left(k_{0}^{*}, k_{1}^{*}\right)-\tilde{\Pi}\left(k^{*}\right)\right) / \partial n>0$ for all $n \in(0, \bar{n})$ and $\partial\left(\tilde{\Pi}_{\text {line }}\left(k_{0}^{*}, k_{1}^{*}\right)-\tilde{\Pi}\left(k^{*}\right)\right) / \partial n \leq 0$ for all $n \geq \bar{n}$, where $\bar{n}=$ $\frac{2(9-\delta)\left(2(1-\bar{c}) \bar{V}^{P}-r\left(1-\delta^{2}\right)(1-\rho) \sigma_{P}^{2}\right)}{r\left(45-51 \delta-\delta^{2}+7 \delta^{3}\right)}$.

- For routine services (i.e., for $\Delta \sigma<0 ; \delta>1$ ):

i) In the range of $\sigma_{P}^{2} \in\left(0, \frac{2(1+\bar{c}) \bar{V}^{P}}{r(\delta-1)(2(1-\rho+\delta)+n \rho+(3 n-2) \delta \rho)}\right]$ we compare the profits $\tilde{\Pi}_{\text {line }}\left(k_{0}^{*}=n, k_{1}^{*}=n / 2\right)$ and $\tilde{\Pi}\left(k^{*}=n\right)$.

ii) In the range of $\sigma_{P}^{2}>\frac{2(1+\bar{c}) \bar{V}^{P}}{r(\delta-1)(2(1-\rho+\delta)+n \rho+(3 n-2) \delta \rho)}$ we compare the profits $\tilde{\Pi}_{\text {line }}\left(k_{0}^{*}=n, k_{1}^{*}=\frac{-2(1+\bar{c}) \bar{V}^{P}+r(\delta-1)(1-\rho+\delta+(2 n-1) \delta \rho) \sigma_{P}^{2}}{r(1-\delta)^{2} \rho \sigma_{P}^{2}}\right)$ and $\tilde{\Pi}\left(k^{*}=n\right)$.

In both cases, it is easy to show that $\tilde{\Pi}_{\text {line }}\left(k_{0}^{*}, k_{1}^{*}\right)-\tilde{\Pi}\left(k^{*}\right)>0$ for any value of $\sigma_{P}^{2}$. After differentiating $\tilde{\Pi}_{\text {line }}\left(k_{0}^{*}, k_{1}^{*}\right)-\tilde{\Pi}\left(k^{*}\right)$ with respect to $n$, we obtain $\partial\left(\tilde{\Pi}_{\text {line }}\left(k_{0}^{*}, k_{1}^{*}\right)-\tilde{\Pi}\left(k^{*}\right)\right) / \partial n>0$ for all $n \in(0, \overline{\bar{n}})$ and $\partial\left(\tilde{\Pi}_{\text {line }}\left(k_{0}^{*}, k_{1}^{*}\right)-\tilde{\Pi}\left(k^{*}\right)\right) / \partial n \leq$ 0 for all $n \geq \overline{\bar{n}}$, where $\overline{\bar{n}}=\frac{\left(1+3 \delta+\sqrt{1+3 \delta^{2}}\right)\left(2(1+\bar{c}) \bar{V}^{P}-r\left(\delta^{2}-1\right)(1-\rho) \sigma_{P}^{2}\right)}{6 r\left(\delta^{2}-1\right) \delta \rho \sigma_{P}^{2}}$. 
With respect to the service length thresholds, we obtain $\bar{n}(\delta=\bar{\delta})-\overline{\bar{n}}(\delta=\overline{\bar{\delta}})>0$, for any $\bar{\delta} \in(0,1)$, and $\overline{\bar{\delta}}>1$.

Observation 1. We consider two competitors, $H$ and $L$, who differ along their capability $\bar{V}^{P}$, where $\bar{V}_{H}^{P}>\bar{V}_{L}^{P}$. We also consider customers to be uniformly distributed according to $\bar{V}^{C}(\theta) \sim \mathcal{U}\left[0, \bar{V}_{H}^{P}\right]$. The are two possible competitive "configurations:" i) the high-capability provider serves the higher end whereas the low-capability provider serves the lower end of the market or, ii) the high-capability provider serves the lower end whereas the low-capability provider servers the higher end of the market. The subsequent analysis reveals that competitive equilibria exist only when the low-capability provider serves the lower-end of the market and the high-capability provider serves the higher-end of the market; hence, we present only the formulation of this competitive scenario. Specifically, in a competitive setting the $\hat{\theta}_{L}$ customer with a capability $\bar{V}^{C}\left(\hat{\theta}_{L}\right) \doteq\left\{\bar{V}^{C}(\theta): \mathbb{E}\left[U\left(\mathcal{P}_{H}, p_{H}\right)\right]-\mathbb{E}\left[U\left(\mathcal{P}_{L}, p_{L}\right)\right]=0\right\}$ is indifferent between the two providers. Similarly, the $\hat{\theta}_{H}$ customer with a capability $\bar{V}^{C}\left(\hat{\theta}_{H}\right) \doteq$ $\left\{\bar{V}^{C}(\theta): \mathbb{E}\left[U\left(\mathcal{P}_{H}, p_{H}\right)\right]-\mathbb{E}[U(\mathcal{P}=\emptyset, p=0)]=0\right\}$ is indifferent between receiving the service from the high-capability provider and entirely self-performing it.

The number of customers that the low-capability provider serves through the $\left(k_{L}, p_{L}\right)$ service is

$$
\begin{aligned}
M_{L}\left(p_{L}, k_{L}, p_{H}, k_{H}\right) \doteq & \frac{\bar{V}^{C}\left(\hat{\theta}_{L}\right)}{\bar{V}^{P}}=\frac{p_{L}-p_{H}+k_{H} \bar{V}_{L}^{P}-k_{L} \bar{V}_{H}^{P}}{k_{H}-k_{L}} \\
& -\frac{r}{2}(1-\delta)\left(1+\left(k_{L}+k_{H}-1\right) \rho+\delta\left(1+\left(2 n-k_{L}-k_{H}-1\right) \rho\right)\right) \sigma_{P}^{2} .
\end{aligned}
$$

The number of customers served through the $\left(k_{H}, p_{H}\right)$ service of the high-capability provider is

$M_{H}\left(p_{L}, k_{L}, p_{H}, k_{H}\right) \doteq \frac{\bar{V}^{C}\left(\hat{\theta}_{H}\right)-\bar{V}^{C}\left(\hat{\theta}_{L}\right)}{\bar{V}^{P}}=\frac{\frac{2\left(k_{L} p_{H}-k_{H}\left(k_{H}\left(\bar{V}_{H}^{P}-\bar{V}_{L}^{P}\right)-p_{L}\right)\right)}{k_{H}\left(k_{H}-k_{L}\right)}+r k_{L}(1-\delta)^{2} \rho \sigma_{P}^{2}}{2 \bar{V}^{P}}$.

The maximization problem of the high-capability provider is given by $\underset{0 \leq k_{H} \leq n, p_{H} \geq 0}{\operatorname{maximize}} \Pi_{H}=$ $\left(p_{H}-k_{H} \bar{c} \bar{V}_{H}^{P}\right) M_{H}$ subject to $M_{H} \in[0,1]$. Similarly, the low-capability provider solves $\underset{0<k_{L} \leq n, p_{L} \geq 0}{\operatorname{maximize}} \Pi_{L}=\left(p_{L}-k_{L} \bar{c} \bar{V}_{L}^{P}\right) M_{L}$ subject to $M_{L} \in[0,1]$.

As in Propositions 3 and 4 , for given $k_{L}, k_{H}$ we derive the prices:

$$
\begin{aligned}
& \tilde{p}_{H}=\frac{k_{H}\left(2\left(k_{L}\left(\left(2 \bar{V}_{H}^{P}+\bar{V}_{L}^{P}\right) \bar{c}+\bar{V}_{H}^{P}\right)-k_{H}\left(2 \bar{V}_{H}^{P}-\bar{V}_{L}^{P}\right)\right)+(1-\delta) r \sigma_{P}^{2}\left(k_{L}-k_{H}\right)\left(-\delta+\rho\left(\delta-(1-\delta) k_{H}-\delta\left(k_{L}+2 n\right)+k_{L}+1\right)-1\right)\right)}{2\left(4 k_{L}-k_{H}\right)}, \\
& \tilde{p}_{L}=\frac{2 k_{H}^{2}\left(\bar{V}_{H}^{P}-\bar{V}_{L}^{P}\right)+4 k_{L}^{2}\left(\bar{V}_{H}^{P}+\bar{c} \bar{V}_{L}^{P}\right)-2 k_{H} k_{L}\left(2 \bar{V}_{L}^{P}-\bar{c} \bar{V}_{H}^{P}\right)-k_{L}\left(k_{L}-k_{H}\right) r(1-\delta)\left(\delta\left(\rho\left(k_{H}+2 k_{L}-4 n+2\right)-2\right)+\rho\left(2-k_{H}-2 k_{L}\right)-2\right) \sigma_{P}^{2}}{2\left(4 k_{L}-k_{H}\right)} .
\end{aligned}
$$


After substituting in $\Pi_{H}$ and $\Pi_{L}$ we obtain:

$\tilde{\Pi}_{H}\left(k_{H} ; k_{L}\right)=\frac{k_{L} k_{H}\left(2 k_{H}\left(\bar{V}_{L}^{P}-(2-\bar{c}) \bar{V}_{H}^{P}\right)+2 k_{L}\left((1-2 \bar{c}) \bar{V}_{H}^{P}+\bar{V}_{L}^{P}\right)-\left(k_{L}-k_{H}\right)(1-\delta)\left(1+\delta-\left(1+k_{L}-k_{H}(1-\delta)+\delta-\left(2 n+k_{L}\right) \delta\right) \rho\right) \sigma_{P}^{2}\right)^{2}}{4\left(4 k_{L}-k_{H}\right)^{2}\left(k_{L}-k_{H}\right) \bar{V}_{H}^{P}}$,

$\tilde{\Pi}_{L}\left(k_{L} ; k_{H}\right)=\frac{\left(2\left(2 k_{L}^{2}+\bar{c} k_{L} k_{H}-k_{H}^{2}\right) \bar{V}_{H}^{P}+2\left(k_{H}-2 k_{L}\right)\left(k+\bar{c} k_{L}\right) V_{L}^{P}+\left(k_{H}-k_{L}\right) k_{L} r(1-\delta)\left(2+\left(k_{H}+2 k_{L}-2\right) \rho+\delta\left(2+\left(4 n-2 k_{L}-k_{H}-2\right) \rho\right)\right) \sigma_{P}^{2}\right)^{2}}{4\left(4 k_{L}-k_{H}\right)^{2}\left(k_{L}-k_{H}\right) \bar{V}_{H}^{P}}$.

The high-capability provider maximizes $\tilde{\Pi}_{H}\left(k_{H} ; k_{L}\right)$ subject to $0 \leq k_{H} \leq n$, $\tilde{M}_{H}\left(k_{H} ; k_{L}\right) \doteq M_{H}\left(k_{H}, p_{H}=\tilde{p}_{H}, p_{L}=\tilde{p}_{L} ; k_{L}\right) \in[0,1]$ (analytical expression available upon request) and the low-capability provider maximizes $\tilde{\Pi}_{L}\left(k_{L} ; k_{H}\right)$ subject to $0 \leq k_{L} \leq n$, $\tilde{M}_{L}\left(k_{L} ; k_{H}\right) \doteq M_{L}\left(k_{L}, p_{L}=\tilde{p}_{L}, p_{H}=\tilde{p}_{H} ; k_{H}\right) \in[0,1]$ (analytical expression available upon request). To determine the competitive equilibria we need to simultaneously maximize the profits of the two providers.

Similar to Proposition 4, the optimal designs are determined by the critical points that satisfy the Kuhn-Tucker conditions. Given the analytical complexity of $\tilde{\Pi}_{H}\left(k_{H} ; k_{L}\right)$ and $\tilde{\Pi}_{L}\left(k_{L} ; k_{H}\right)$ we find the solutions to the Kuhn-Tucker conditions via numerical methods and for different values of $n, \delta, r, \sigma_{P}^{2}, \rho, \bar{c}$, and $\bar{V}_{H}^{P}$ (e.g., see Figure 9 for an example of such values used). The qualitative insights (see Figure 9) are not sensitive to different value combinations for the service parameters. 


\section{References}

Ba, S., J. Stallaert, Z. Zhang. 2010. Balancing IT with the Human Touch: Optimal Investment in IT-Based Customer Service. Inf. Syst. Res. 21(3) 423-442.

Basu, A., S. Bhaskaran. 2018. An Economic Analysis of Customer Co-design. Forthcoming in Inf. Syst. Res.

Bellos, I., S. Kavadias. 2017. Service Design for a Holistic Customer Experience: A Process Perspective. Working Paper.

Berry, L., L. Carbone, S. Haeckel. 2002. Managing the Total Customer Experience. MIT Sloan Manage. Rev. $43(3)$ 85-89.

Bitner, M., S. Brown, M. Meuter. 2000. Technology Infusion in Service Encounters. J. Acad. Marketing Sci. 28(1) 138-149.

Brown, T. 2008. Design Thinking. Harvard Bus. Rev. 86(6) 84.

Buell, R., D. Campbell, F. Frei. 2010. Are Self-Service Customers Satisfied or Stuck? Production and Oper. Management 19(6) 679-697.

Campbell, D., F. Frei. 2010. Cost Structure, Customer Profitability, and Retention Implications of SelfService Distribution Channels: Evidence from Customer Behavior in an Online Banking Channel. Management Sci. 56(1) 4-24.

Chowhound. 2011. When Restaurants Refuse Substitutions. http://www.chowhound.com/food-news/ 84285/when-restaurants-refuse-substitutions/. Accessed July 15, 2018.

Dabholkar, P. 1991. Using Technology-Based Self-Service Options to Improve Perceived Service Quality. AMA Summer Educators Conf. Proc. 534-535.

Dabholkar, P. 1996. Consumer Evaluations of New Technology-Based Self-Service Options: An Investigation of Alternative Models of Service Quality. Internat. J. of Res. Marketing 13(1) 29-51.

Demirezen, E., S. Kumar, B. Shetty. 2016. Managing Co-Creation in Information Technology Projects: A Differential Games Approach. Inf. Syst. Res. 27(3) 517-537.

Franke, N., M. Schreier, U. Kaiser. 2010. The "I designed it myself" Effect in Mass Customization. Management Sci. 56(1) 125-140.

Frei, F., A. Morriss. 2012. Uncommon Service. Harvard Business Press.

Froehle, C., A. Roth. 2004. New Measurement Scales for Evaluating Perceptions Of The Technology-Mediated Customer Service Experience. J. Oper. Management 22(1) 1-21.

Goldfarb, A., R. McDevitt, S. Samila, B. Silverman. 2015. The Effect of Social Interaction on Economic Transactions: Evidence from Changes in Two Retail Formats. Management Sci. 61(12) 2963-2981.

Karmarkar, U., G. Roels. 2015. An Analytical Framework for Value Co-production in Services. Service Sci. 7 (3) $163-180$. 
Krishnan, V., S. Gupta. 2001. Appropriateness and Impact of Platform-based Product Development. Management Sci. 47(1) 52-68.

Krishnan, V., W. Zhu. 2006. Designing a Family of Development-Intensive Products. Management Sci. $\mathbf{5 2}(6) 813-825$.

Kumar, A., R. Telang. 2012. Does The Web Reduce Customer Service Cost? Empirical Evidence From A Call Center. Inf. Syst. Res. 23(3-part-1) 721-737.

Lacourbe, P., C. Loch, S. Kavadias. 2009. Product Positioning in a Two-Dimensional Market Space. Production and Oper. Management 18(3) 315-332.

Leonard, D., J.F. Rayport. 1997. Spark Innovation through Empathic Design. Harvard Bus. Rev. 75 102-115.

Markowitz, H. 1952. Portfolio Selection. J. Finance 7(1) 77-91.

Moon, Y., F. Frei. 2000. Exploding the Self-Service Myth. Harvard Bus. Rev. 78(3) 26-27.

Norton, M., D. Mochon, D. Ariely. 2012. The IKEA Effect: When Labor Leads to Love. J. Consum. Psychol. $22453-460$.

Prahalad, C., V. Ramaswamy. 2000. Co-opting Customer Competence. Harvard Bus. Rev. 78(1) 79-90.

Roels, G., U. Karmarkar, S. Carr. 2010. Contracting for Collaborative Services. Management Sci. 56(5) $849-863$.

Rousseeuw, P., G. Molenberghs. 1994. The Shape of Correlation Matrices. The American Statistician 48(4) 276-279.

Sampson, S., C. Froehle. 2006. Foundations and Implications of a Proposed Unified Services Theory. Production and Oper. Management 15(2) 329.

Sasser, W. 1976. Match Supply and Demand in Service Industries. Harvard Bus. Rev. 54(6) 133-140.

Scherer, A., N. Wünderlich, F. von Wangenheim. 2015. The Value of Self-Service: Long-Term Effects of Technology-Based Self-Service Usage on Customer Retention. Manag. Inf. Syst. Q. 39(1) 177-200.

Soteriou, A., G. Hadjinicola. 1999. Resource Allocation to Improve Service Quality Perceptions in Multistage Service Systems. Production and Oper. Management 8(3) 221-239.

The Wall Street Journal. 2013. Humans 1, Robots 0. https://www.wsj.com/articles/ humans-1-robots-0-1381098947. Accessed July 15, 2018.

The Wall Street Journal. 2016. Panasonic Takes Item Out of Bagging Area: Human. http://www.wsj . com/articles/panasonic-takes-item-out-of-bagging-area-human-1481542493. Accessed July $15,2018$.

Thomke, S., E. Von Hippel. 2002. Customers as Innovators: A New Way to Create Value. Harvard Bus. Rev. 80(4) 74-85.

Varian, H. 1992. Microeconomic Analysis. Norton \& Company, Inc. 
WebMD. 2018. Home Blood Glucose Test. http://www. webmd.com/diabetes/home-blood-glucose-test. Accessed July 15, 2018.

White, S., R. Badinelli. 2012. A Model for Efficiency-Based Resource Integration in Services. Eur. J. Oper. Res. $217(2)$ 439-447.

Xue, M., J. Field. 2008. Service Coproduction with Information Stickiness and Incomplete Contracts: Implications for Consulting Services Design. Production and Oper. Management 17(3) 357-372.

Zeithaml, V., A. Parasuraman, L. Berry. 1985. Problems and Strategies in Services Marketing. J. Marketing 49(2) $33-46$. 